\title{
Numerical Investigation of Quasi-sessile Droplet Absorption into Wound Dressing Capillaries \\ M. Andredaki ${ }^{1,2}$, A. Georgoulas ${ }^{1}$, M. Marengo ${ }^{1,2}$ \\ ${ }^{1}$ Advanced Engineering Centre, School of Computing Engineering and Mathematics, University of Brighton, Brighton, BN2 4GJ, UK. \\ ${ }^{2}$ Centre for Regenerative Medicine and Devices, School of Pharmacy and Biomolecular Sciences, University of Brighton, Brighton BN2 4GJ, UK.
}

\begin{abstract}
*Author to whom correspondence should be addressed:m.andredaki@ brighton.ac.uk
Abstract

The key concept in wound dressing design and development is the fact that keeping a wound moist accelerates healing. Therefore, the selection of the appropriate wound dressing type is vital. The absorption of wound exudate by wound dressings can be considered as a microfluidic phenomenon that can be investigated either by performing high resolution laboratory experiments or by utilising high resolution Computational Fluid Dynamics (CFD) numerical simulations. As an initial step, in the present paper, the effects of the pore size (wound dressing porosity), the liquid (wound exudate) viscosity and the initial droplet diameter, are numerically investigated, using a simplified analogue of the phenomenon that consists of a quasi-sessile droplet, being absorbed by a single cylindrical pore. For this purpose, an enhanced Volume Of Fluid (VOF) model, developed in the general context of OpenFOAM, is validated and applied. It is found that distinct droplet absorption rates exist with specific relationships derived, using best-fit lines, that can predict the absorption rates for particular values of pore size and liquid viscosity. For the examined Eo and Oh number ranges $(0.0015<$ Eo $<0.15$ and $0.0035<\mathrm{Oh}<0.095)$, these distinct droplet absorption rates are directly linked with four different droplet evolution regimes that are grouped in a well-defined flow map. Finally, it is shown that the resulting liquid absorption rates are not significantly affected by the initial droplet diameter and that an appropriate wound dressing porosity can be selected by an estimation of the wound exudate physical properties.
\end{abstract}

\section{Keywords}

Wound dressing, Bio-fluids, Bioengineering, Droplet absorption, Porous surfaces, OpenFOAM

\section{Introduction}

Wound exudate plays a significant role in wound healing procedures. The presence of infection increases the viscosity of wound exudate, due to the changes in bacterial load and protein content. Therefore, the selection of the appropriate type of wound dressing with respect to the exudate's viscosity is vital, in order to avoid having higher viscosity exudates within infected wounds remaining on the wound surface for longer than they should. However, researchers and wound dressing companies, commonly test absorption of wound dressings, applying a protocol (BS EN 13726-1:2002) that uses a specific serum type solution, without taking into consideration the different types of wound exudates [1]. The main challenge for clinicians is to create an environment at the wound bed that optimises the wound healing process. The absorption of wound exudate by wound dressings is a microfluidic phenomenon that can be investigated performing either high resolution laboratory experiments, usually expensive and difficult to implement, or by utilising high resolution Computational Fluid Dynamics (CFD) based numerical simulations.

One of the most significant contributions in wound management that became a key parameter in the design and development of wound dressings was the work by Winter [2], who argued that keeping a wound moist accelerates the healing process. Exudate consists mainly of water, but it also contains electrolytes, nutrients, proteins, inflammatory mediators, protein-digesting enzymes, such as matrix metalloproteinases, growth factors and waste products, as well as cells such as neutrophils, macrophages and platelets [3] and is produced during the inflammatory and pro-liferative stages of the healing process. However, the volume that is produced varies not only among different wound 
types, depending on the wound size, origin and location, but it is also related to the stage of the healing process.

According to Ovington [4], chronic or nonhealing wounds (venous leg ulcers or wounds in a setting of lymphedema) may be associated with very high exudate levels. In this case, the dressing must be able to manage the exudate by absorption to establish optimal tissue moisture levels. If the wound tissues are adequately moist with minimal exudate production, then the dressing should be capable of maintaining the tissue hydration status without too much absorption that could desiccate the wound. Alternatively, if tissue moisture levels are already depleted, the dressing must be able to restore optimal tissue hydration by donating moisture to the wound. Currently more than 3000 types of dressings are available in the market, making it challenging to the physicians to address all aspects of wound care [5]. This implies the lack of full understanding of wound care and management [6].

Early testing of absorbency and dressing specifications were described by Piskozub [7] and Thomas et al. [8]. As more sophisticated dressings were developed, new standards and test systems were required to prove that these new materials performed a specific function in consistent and reproducible ways. Thomas [9] discussed the need for dressing standards, described how these have evolved, and outlined test methods that could be used to assess key aspects of performance in many different available products.

The best method of evaluating a wound dressing is an in vivo clinical trial. However, too few have been undertaken and published ([10]-[13]). This is also the case with quantitative, comparative in vitro data [14]-[16]. Further research into quantitative methods of evaluating these products and more clinical trials are urgently needed, especially to assist practitioners with selection of appropriate wound dressings.

According to White and Cutting [17], effective clinical management of exuding wounds depends on accurate assessment of the volume and viscosity of exudate. However, there are no investigations in the literature up to date that explicitly examine the effect of exudate viscosity and there is also a lack of qualitative information regarding exudate viscosity value ranges. Only qualitative references to exudate types being watery, viscous or sticky exist (e.g. [18], [19]).

As mentioned previously, the absorption of wound exudate by wound dressings is a microfluidic phenomenon. According to the Authors' best knowledge, there are no previous experimental and/or numerical investigations directly examining the absorption of wound exudate into wound dressing capillaries. Therefore, in the following paragraphs indirectly related studies on liquid droplet absorption into porous substrates are reviewed.

Navaz et al. [20] investigated numerically the primary and secondary spread of sessile droplets into a porous substrate, utilizing a continuum approach for liquid- and gas-phases. The governing equations were discretized by the finite difference method and solutions for both phases are obtained by marching in time using the fourth order Runge-Kutta integration algorithm. This type of spread is a purely momentum-driven process that is caused by gradients both in capillary pressure and in saturation. They developed a methodology for finding the capillary pressure function for sessile droplets. This approach was based on experimental data for a liquid/porous medium pair, and using universal, non-dimensional curves. Similar solutions were generated by the continuum approach and validated using experimental results. The model showed qualitative and quantitative agreement with experimental data. Although the focus of their work was to understand the interaction of chemical warfare agents with porous media, the approaches are universal and can be applied to determining the spread of any liquid into a porous material.

Markicevic et al. [21] investigated numerically the infiltration of a sessile droplet into a homogeneous porous medium for a constant droplet base radius case, where the porous medium was represented as a capillary network consisting of pores and throats. They carried out a parametric study, varying (i) the liquid viscosity and surface tension, (ii) droplet volume and base radius, and (iii) porous medium porosity and permeability. The droplet infiltration time, and the imprint shape that was given with two spheroid half-axes were calculated. Dimensionless analysis was utilized to correlate the droplet infiltration parameters from which master curves for the droplet infiltration time 
and the droplet imprint shape were obtained. Using the infiltration time correlation, both numerical and experimental results showed a linear behaviour.

In their paper, Frank and Perré [22] presented an investigation into drop spreading at the surface of a porous substrate, using a pore-level numerical model based on a Lattice-Boltzmann scheme. Two contact angles were tested, and two porosity values were investigated and compared with a non-porous surface. The numerical results shown a power-law evolution of the wetted zone radius with time. Both the exponent and the pre-factor were decreasing with increasing porosity. The evolution in time of the droplet height emerged from a competition between pure spreading and bulk capillary imbibition within the porous medium.

Denesuk et al. [23] developed a model for the infiltration of finite size liquid droplets into porous materials, including the effects of the instantaneous droplet size on the number of pores in contact with the droplet, investigating the time dependence of the droplet radius under two limiting case assumptions concerning the kinetic behaviour of the droplet as its volume was depleted. Their results were tested against experimental data of silicone liquids on a soda-lime-silicate porous solid.

In a recent study, Sadeghi et al. [24] implemented a three-dimensional multiphase lattice Boltzmann model, validated against the analytical solution of Young's and Laplace's laws, in order to investigate the spontaneous phase transport in complex porous media and the effects of several geometrical and flow parameters such as porosity, density ratio, Reynolds number, Weber number, Froude number and contact angle. A parametric study of the influence of main non-dimensional parameters upon the impact of liquid drops on permeable surface was also performed.

Choi et al. [25] presented a level-set method for computation of droplet impact and penetration into a porous medium. The volume averaged conservation equations of mass and momentum were employed for the porous region, including the effects of porosity and drag force caused by the porous solid matrix, being coupled to the conservation equations in the external fluid region through the matching conditions of velocity and stress on the porous surface. They conducted simulations to investigate the effects of initial droplet radius, impact velocity, contact angles, particle size and porosity on the droplet spreading and penetration. A simple analytical formulation was also developed for the initial droplet penetration depth and compared with the numerical results.

To determine the influence of the fabric on droplet spreading using thin fabric meshes, de Goede et al. [26] measured the droplet spreading ratio on fabric with and without an underlying substrate using a high-speed camera. For fabrics without a substrate, the droplet spreading ratio was reduced as the fabric penetration by the liquid reduced the droplet volume spreading on top of the fabric. Using entropic lattice Boltzmann simulations, they found that the lower droplet spreading ratio on fabrics, both with and without a substrate, is due to an increase of viscous losses inside the droplet during spreading. Comparing droplet impact of blood with its Newtonian counterpart, they also showed that for spreading on fabrics, just like on smooth surfaces, blood can be approximated as a Newtonian fluid.

In their study, Koponen et al [27] introduced a modification of Kozeny and Carman permeability equation [28]-[30] to include the effect of effective porosity. An analytical expression for the specific surface area of a system constructed of randomly placed identical obstacles with unrestricted overlap was derived, and a lattice-gas cellular automaton method was used to simulate the dependence on porosity of permeability, tortuosity, and effective porosity for a flow of Newtonian uncompressible fluid in a two- dimensional porous substance.

Yu and Cheng [31] developed a fractal permeability model for bi-dispersed porous media, based on the fractal characteristics of the pores, validated using experimental data. Their model was found to be a function of the tortuosity fractal dimension, pore area fractal dimension, sizes of particles and clusters, micro-porosity inside clusters, and the effective porosity of a medium. The pore area fractal dimension and the tortuosity fractal dimension of the porous samples were determined by the box counting method.

An initial approach provided by experiments performed with the elementary geometry of a substrate with a single capillary has been presented in [32], [33]. The work by Kogan et al [32] consisted of experimental studies, using a high speed camera, and model calculations, using the 
Bosanquet capillary flow equation. These studies were performed investigating the capillary flow of a viscous liquid caused by droplet impact on a porous surface. A series of experiments was conducted in which the dynamics of capillary flow was monitored under the conditions of single droplet impact on a transparent block containing a single capillary. Capillaries having $0.1,0.3$, and $0.5 \mathrm{~mm}$ in diameter were used in their work; droplets were generated using DI water and a 0.1 percent solution of Triton X-100 surfactant as the test liquids. Delbos et al. [33] investigated experimentally how the impregnation of porous media can be forced using the initial kinetic energy of an impacting drop. They focused on the scale of a single pore - either hydrophilic or hydrophobic - and thus studied the impact of a single drop falling on vertical cylindrical capillary tubes. They observed different limit regimes and they determined the critical speeds for the different limit regimes, they had observed, and obtained a full phase-diagram for their observations. They also stressed the characteristics of impregnating slugs namely their volume and their motion within the pores.

The experiments by Delbos et al. [33] were reproduced numerically by Ding and Theofanous [34], who then generalized them by mapping numerical results in the $\mathrm{We}-\mathrm{Re}_{\mathrm{r}}$ plane. Interesting phenomena were observed above the substrate and inside the capillary, though the related mechanisms were shown to be different. Inertia appeared to be crucial in all these events: inertia from the conversion of surface energy was more important in droplet ejection above the substrate, while sufficient impact inertia was a necessity for droplet ejection inside the capillary. The significance of impact inertia in the slug formation was reflected by a spatial anchoring of stagnation region with time. As a result, the amounts of liquid inside the slug were found to come from an upright cylinder above the capillary. This was in agreement with the hypothesis of Delbos et al.[33], but the radius of the cylinder was $30 \%$ greater than the capillary radius. Inertia and contact-angle hysteresis were shown to be the most important factors in the transition of penetration regimes; the latter had an especially significant effect on the transition to the regime of partial penetration as a slug.

All these previous investigations are divided into two main categories. The first one examines the spreading of a sessile drop above the porous medium, without focusing on the actual phenomena that happen within the porous media, due to the complexity of the pore structure. On the other hand, the second branch of investigations examine a single pore, in order to be able to study and quantify the underpinned characteristics and mechanisms within the pore, but using impacting droplets, where the initial momentum of the droplet affects significantly the liquid penetration characteristics within the pore. Therefore, the absorption characteristics cannot be isolated, identified and quantified. In the present paper, since the ultimate aim is to understand how the absorption characteristics are changing for different fluid viscosities, which would be of direct interest for wound dressing applications that constitute the main motivation for this study, non-impacting, quasi-sessile droplets that are absorbed by capillary action within a single pore, are addressed for the first time, using numerical simulations. For this purpose, an enhanced, user-defined, Volume Of Fluid (VOF) model that has been developed by the authors in OpenFOAM is used. The examined pore sizes have been selected in order to fall within typical pore size ranges reported in the literature for various types of wound dressings [35], [36]. Finally, with respect to the selected fluid properties, the fluid viscosity is varied from typical water viscosity values up to eight times higher in order to cover potential viscosity values for various types of wound exudates. As mentioned previously, there is a lack of qualitative information in the literature for exudate viscosity values. Therefore, the proposed range of viscosities was selected in order to account for watery up to more viscous exudates that are quantitatively referred in the literature ([18], [19]). Since the specific gravity of exudate is reported as been greater than 1.020 [19], the fluid density value in the present investigation was chosen to be at this low limit, being close to the density of water at ambient conditions. As for the surface tension the value of water at ambient conditions was also selected, since there is no information at all in the literature for surface tension values of wound exudate.

As mentioned previously, in order to isolate the absorption phenomenon quasi-sessile droplet depositions are considered in the present numerical investigation. Therefore, a zero-impact velocity is considered in all cases. For this purpose, Ohnesorge (Oh) and Eötvos (Eo) numbers are utilised to describe the investigated phenomenon. The examined dimensionless numbers ranges are $0.0015<$ Eo 
$<0.15$ and $0.0035<\mathrm{Oh}<0.095$ and these result due to the selected pore sizes and fluid viscosity ranges, as discussed previously, in order to be relevant to cases of wound exudate absorption into wound dressing capillaries. At this point, it should be mentioned that in future applications, also the concepts of liquid film instead of a drop as well as of multiple pores and pore networks worth to be examined to further extend the results of the present investigation, bringing them closer to the targeted application.

\section{Mathematical framework}

From a fluid-dynamics point of view, the aforementioned phenomenon is governed by the interaction of two immiscible fluids, with a clearly defined interface between them; Ambient air that pre-exists in the pore structure of the wound dressing at the moment of application and the liquid exudate that leaks out from the affected wound, usually with a relatively low flow rate. Hence, the actual resulting absorption rate is an interplay of the following mechanisms:

- interface dynamics between a gaseous and a liquid fluid phase

- capillary action within micro-passages

- wettability

For this purpose, an improved Volume Of Fluid (VOF) based model, which has been previously implemented in OpenFOAM CFD Toolbox (an open source CFD software), is utilised. In more detail, all the numerical simulations of the present work were performed with the finite volumebased CFD code OpenFOAM (version 2.2.1), using a user-enhanced version of its original VOFbased solver "interFoam". For pressure-velocity coupling, the PISO (Pressure-Implicit with Splitting of Operators) scheme is applied. The transient terms in the equations are discretised using a second order, bounded, implicit scheme (Euler). The calculation time step is controlled by setting the maximum Courant number to 0.2. With this adaptive time-stepping technique, the time step is automatically varied from approximately $10^{-9}$ to $10^{-6}$ seconds for the overall simulation cases that are presented in the present paper. The gradient terms are discretised using a second-order, Gaussian integration with linear interpolation (Gauss linear). For the divergence terms, different discretisation schemes are applied for each term in the equations. In more detail, the convection term of Equation (2) is discretised using a "Gauss upwind" scheme. The $\nabla \cdot(\alpha \vec{U})$ term of Equation (3) is discretised using the "Gauss vanLeer" scheme, while the $\nabla \cdot\left(\alpha(1-\alpha) U_{r}\right)$ term is discretised using the "Gauss interfaceCompression" scheme which ensures the boundedness of the calculated volume fraction field. Finally, all Laplacian terms are discretised using the "Gauss Linear Corrected" scheme. Further details on the proposed discretisation schemes can be found in OpenFOAM documentation [37]. It should be mentioned that this was the optimum combination of discretisation schemes in order to maintain a balance between accuracy, convergence and numerical stability during the computations.

With the VOF approach, the transport equation for the volume fraction, $\alpha$, of the liquid phase is solved simultaneously with a single set of continuity and Navier-Stokes equations for the whole flow field. The corresponding volume fraction of the gas phase is simply calculated as $(1-\alpha)$. The main underlying assumptions are that the two fluids are Newtonian, incompressible, and immiscible. The governing equations can be written as,

$$
\begin{gathered}
\nabla \cdot \vec{U}=0 \\
\frac{\partial \rho_{b} \vec{U}}{\partial t}+\nabla \cdot\left(\rho_{b} \vec{U} \vec{U}\right)=-\nabla p+\nabla \cdot \mu_{b}\left(\nabla \vec{U}+\nabla U^{T}\right)+\rho_{b} f+F_{s} \\
\frac{\partial a}{\partial t}+\nabla \cdot(\alpha \vec{U})-\nabla \cdot\left(\alpha(1-\alpha) U_{r}\right)=0
\end{gathered}
$$

where the bulk fluid properties $\left(\rho_{b}, \mu_{b}\right)$ are calculated as weighted averages of the liquid $\left(\rho_{l}, \mu_{l}\right)$ and gaseous $\left(\rho_{g}, \mu_{g}\right)$ phase properties as follows,

$$
\begin{gathered}
\rho_{b}=\rho_{l} \alpha+\rho_{g}(1-\alpha) \\
\mu_{b}=\mu_{l} \alpha+\mu_{g}(1-\alpha)
\end{gathered}
$$


The surface tension force is modelled as a volumetric force using the Continuum Surface Force (CSF) method by Brackbill et al. [38] applying the following equation

$$
\begin{gathered}
F_{S}=\gamma \mathrm{k}(\nabla \alpha) \\
\mathrm{K}=\nabla \cdot\left(\frac{\nabla \tilde{\alpha}}{|\nabla \tilde{\alpha}|}\right)
\end{gathered}
$$

where, $\gamma$ is the surface tension coefficient and $\mathrm{k}$ is the curvature of the interface. As mentioned previously the utilized numerical framework constitutes an enhanced version [39] of the original VOF-based solver of OpenFOAM [37], that suppresses numerical artefacts of the original model, known as "spurious currents". The proposed enhancement involves the calculation of the interface curvature $\mathrm{K}$ using the smoothed volume fraction values $\widetilde{\alpha}$, which are obtained from the initially calculated $\alpha$ field, smoothing it over a finite region near the interface. All other equations are using the initially calculated (non-smoothed) volume fraction values of $\alpha$.

Furthermore, in order to accurately account for wettability effects, Kistler's Dynamic Contact Angle (DCA) model [40], has been also implemented in the proposed VOF solver which calculates the DCA, $\theta_{d}$, using the Hoffman function, $f_{H o f f}$, as follows:

$$
\theta_{d}=f_{\text {Hoff }}\left[C_{a}+f_{\text {Hoff }}^{-1}\left(\theta_{\varepsilon}\right)\right]
$$

where, $\theta_{\epsilon}$ is the equilibrium contact angle. The capillary number, $C_{a}$, is calculated with respect to the spreading velocity of the contact line a $U_{\mathrm{CL}}$ and $f_{H o f f}^{-1}$ is the inverse function of "Hoffman's" empirical formulae which is calculated as shown below,

where $\mathrm{x}$ is equal to:

$$
f_{\text {Hoff }}=\operatorname{acos}\left[1-2 \tanh \left(5.16\left(\frac{x}{1+1.31 x^{0.99}}\right)^{0.706}\right)\right]
$$

$$
x=f_{H}\left[C a+f_{H}^{-1}\left(\theta_{\mathrm{eq}}\right)\right]
$$

The capillary number is defined as

$$
\text { Ca }=\frac{\mu u_{\text {cline }}}{\gamma}
$$

The equilibrium angle $\theta_{\varepsilon}$ is replaced by either a limiting advancing $\left(\theta_{a}\right)$ or receding contact angle $\left(\theta_{r}\right)$, depending on the sign of the velocity vector at the contact line.

Further details on the development and validation of the proposed numerical modelling framework can be found in [39], [41] and [42].

\section{Numerical model validation}

In order to further validate the numerical model, for cases more relevant to the present investigation, its predictions have been compared against the experimental results of the work of Delbos et al. [33], for the case of a water droplet with a diameter of $2.5 \mathrm{~mm}$ that impregnates into capillary glass tubes that have been plasma treated (hydrophilic surfaces). The length of the considered tubes is $5 \mathrm{~cm}$ long in each case with inner diameters of $0.20 \mathrm{~mm}, 0.44 \mathrm{~mm}$ and $1 \mathrm{~mm}$. In order to reproduce the same conditions as in the proposed experiments, a dynamic viscosity $\mu=1$ mPas, a density of $\rho=1000 \mathrm{~kg} / \mathrm{m}^{3}$ and a surface tension $\gamma=70 \mathrm{mN} / \mathrm{m}$ were used in the numerical simulations for the liquid phase. As for the pre-existing gaseous phase in the considered domains, air properties at standard conditions were used. For the wettability characteristics, a static contact angle of 30 degrees and a contact angle hysteresis $\cos \theta_{r}-\cos \theta_{a}$ of 0.3 were used in the simulations. The corresponding impact velocities for these three cases were $0.15 \mathrm{~m} / \mathrm{s}, 1.00 \mathrm{~m} / \mathrm{s}$ and $1.30 \mathrm{~m} / \mathrm{s}$, respectively. It should be highlighted at this point that due to lack of literature available experimental, 
quasi-sessile droplet depositions on single cylindrical pores, these impact cases are considered instead, for validating the utilised numerical simulation framework.

In the work of Antonini et al. [43], the values of $\theta_{a}$ and $\theta_{r}$ for water and glass have been measured experimentally to be equal to 48 and (less than) 5 degrees, respectively. Since these values satisfy the relationship among the three contact angles (constant, advancing and receding) that is found and reported in the work of Miyama et al. [44] (please see Equation 12 below), for the case of constant (equilibrium) contact angle of $\theta_{c}=30^{\circ}$, they are safely adopted as inputs for the numerical simulations of the present investigation:

$$
\cos \theta_{c}=\frac{\cos \theta_{r}+\cos \theta_{a}}{2}
$$

The computational domain, the computational mesh and the applied boundary conditions are shown in Figure 1. Due to the axial symmetry of the investigated phenomenon, 2D axisymmetric simulations are performed. Therefore, a wedge type geometry is constructed for the computational domain, representing a $5^{\circ}$ wedge of the corresponding full 3D domain. A uniform, structured mesh is utilised. The results from the mesh independency study that are presented in the following section, indicated the use of a cell size of $2 \mu \mathrm{m}$. A qualitative comparison of the numerical simulation predictions for successive time instances in relation to the corresponding experimental data [33], are depicted in Figures 2, 3 and 4, for the cases with pore radius $0.10 \mathrm{~mm}, 0.22 \mathrm{~mm}$ and $0.50 \mathrm{~mm}$, respectively. As it is can be seen, there is a very good agreement between the numerical predictions and the experimental snapshots regarding the spatial and temporal evolution of the spreading as well as the penetrating droplet parts, in each case. Furthermore, it is evident that the numerical simulations successfully capture the development of the different two-phase flow regimes within the cylindrical tubes, in each case, that also Delbos et al. [33], have observed experimentally.

In more detail, for pore radius $0.10 \mathrm{~mm}$ (Figure 2), both in the numerical predictions as well as in the experimental snapshots, a small part of the impacting drop penetrates the cylindrical pore, filling up the entire pore cross section, remaining connected with the spreading drop above the pore, for all stages. The penetration depth gradually increases with time. For the case where the pore radius is $0.22 \mathrm{~mm}$ (Figure 3), both in the numerical predictions as well as in the experimental snapshots, when the droplet at the top of the capillary reaches its maximum spread a temporary cleaving is observed and then at the recoiling stage of the droplet, liquid penetration resumes, causing the entrapment of an elongated air plug (dry patch) with the overall liquid column. Finally, for the case where the pore size is $r_{t}=0.50 \mathrm{~mm}$ (Figure 4), both in the numerical predictions as well as in the experimental snapshots, a slug of liquid that is disconnected from the rest of the drop is formed, due to a permanent cleaving, after the initial liquid penetration. For clarification purposes it should be mentioned that in all three figures (Figure 2, 3 and 4), as it is indicated with the red arrows in Figure 2 , in the experimental snapshots, below the cylindrical pore inlet, the colour representation for liquid and gas phases is reversed, with dark colour representing the gaseous phase and light colour representing the liquid phase. Above the pore inlet, the dark colour represents the liquid phase and the light colour the surrounding air. For the numerical simulation results however, a common colour code is used both above and below the pore inlet, with the dark colour representing the liquid phase and the light colour representing the gaseous phase. 


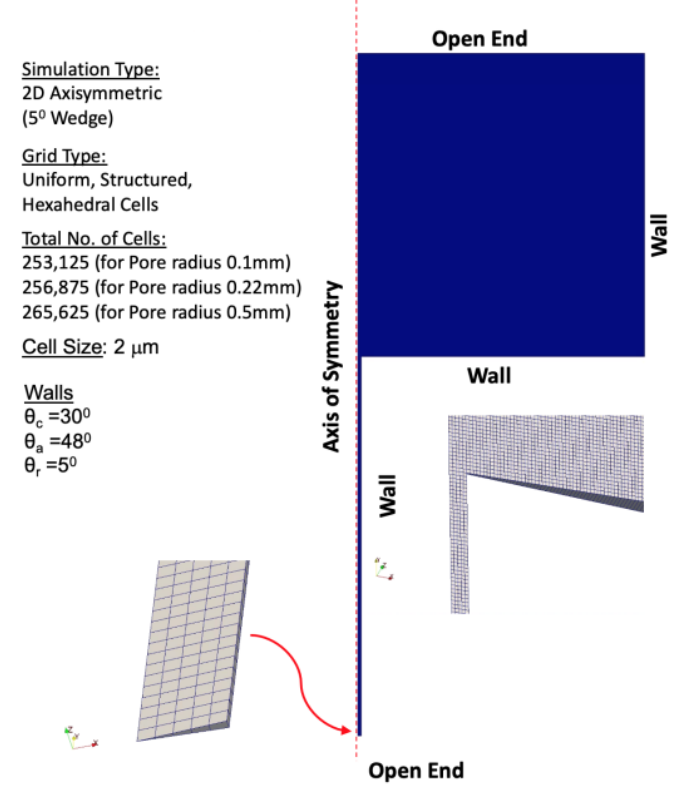

Figure 1. Computational domain, computational mesh and boundary conditions.
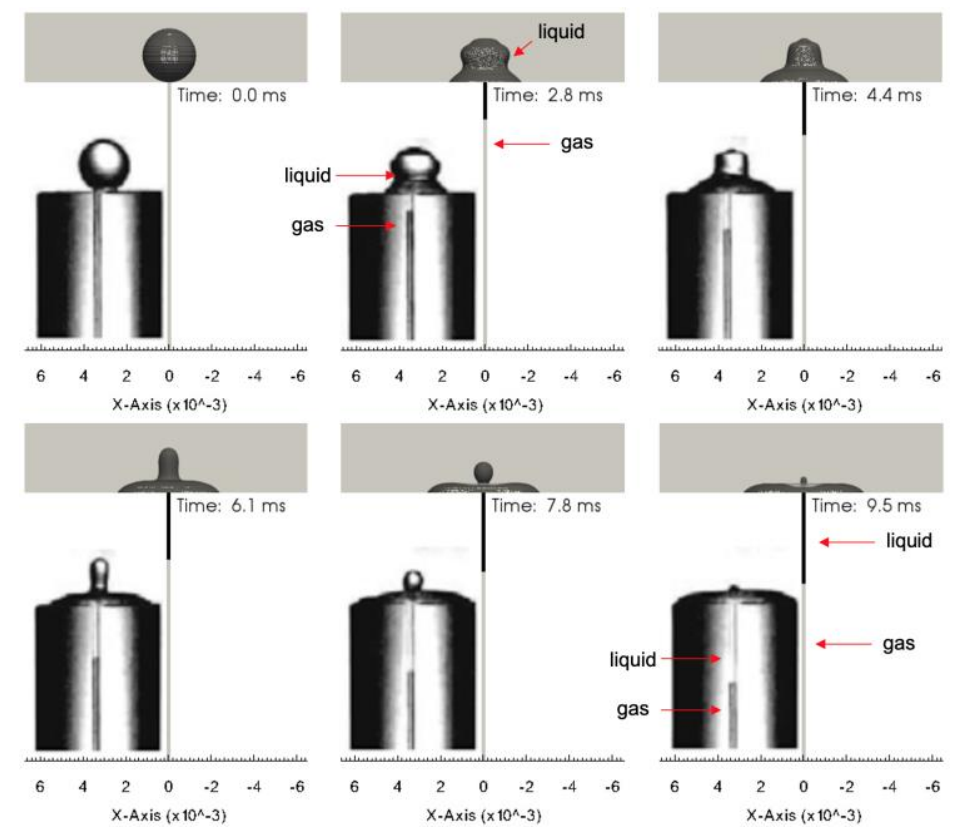

Figure 2. The results of the simulations at different time instances and the comparison with the experiments [33], for the case with tube radius $r_{t}=0.10 \mathrm{~mm}$ and impact velocity $v_{i}=0.15 \mathrm{~m} / \mathrm{s}$. A small part of the impacting drop penetrates the cylindrical pore filling up the entire pore cross section, while remaining connected with the spreading drop above the pore, for all stages. The penetration depth gradually increases with time. 


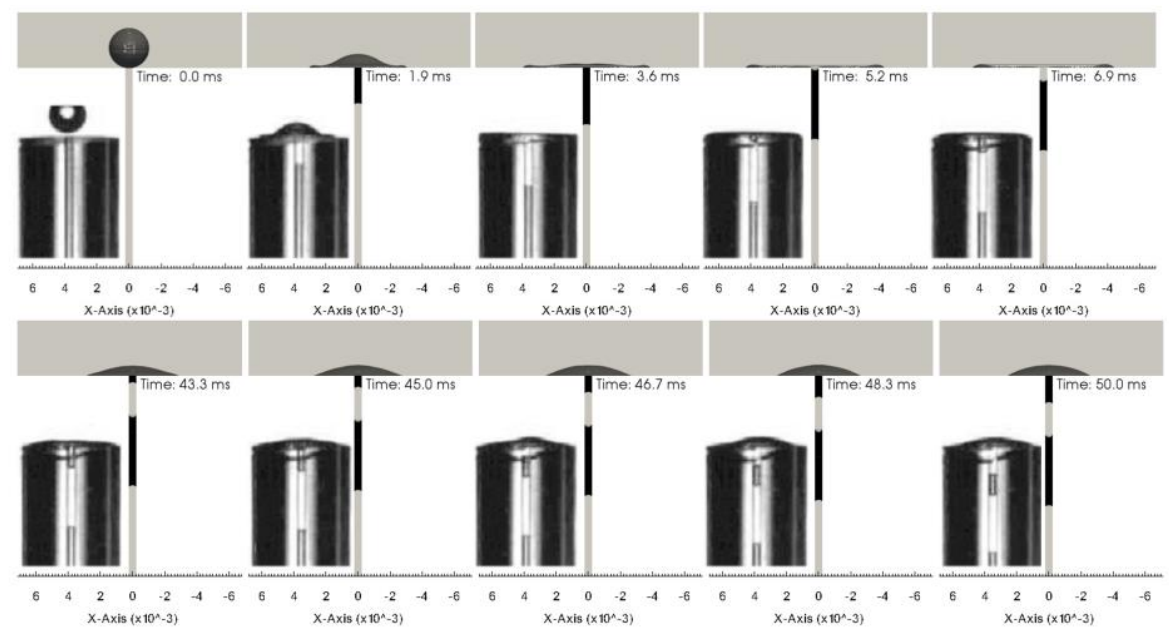

Figure 3. The results of the simulations at different time instances and the comparison with the experiments [33], for the case with tube radius $r_{t}=0.22 \mathrm{~mm}$ and impact velocity $v_{i}=1.00 \mathrm{~m} / \mathrm{s}$. When the droplet at the top of the capillary reaches its maximum spread a temporary cleaving is observed and then at the recoiling stage of the droplet, liquid penetration resumes, causing the entrapment of an elongated air plug (dry patch) with the overall liquid column.

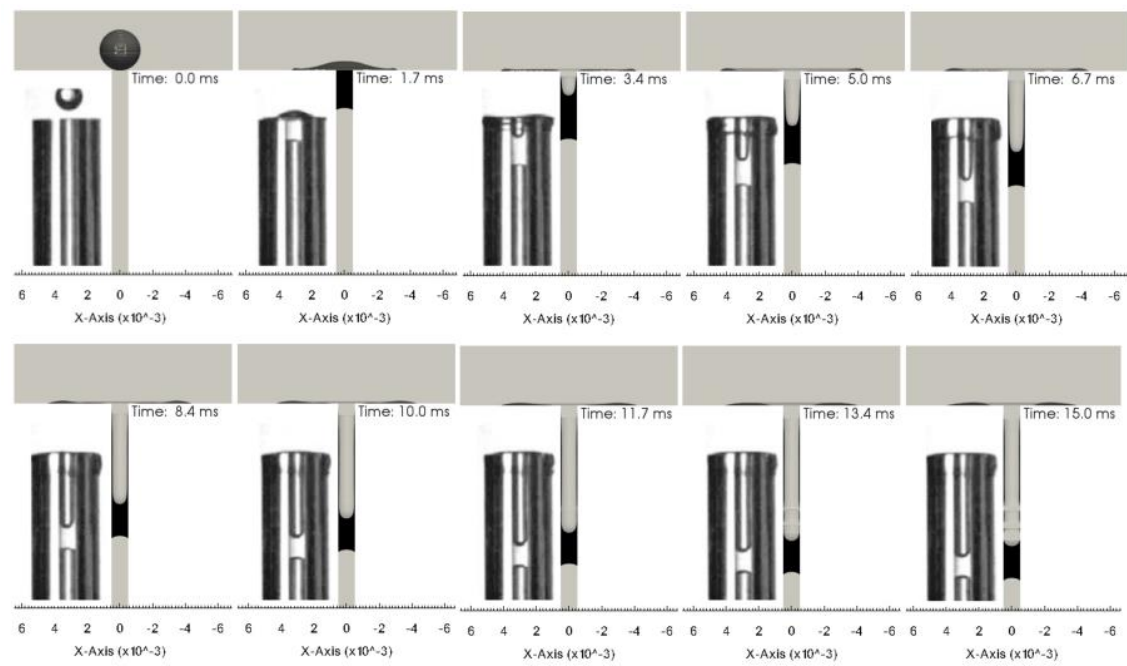

Figure 4. The results of the simulations at different time frames and the comparison with the experiments [33], for the case with tube radius $r_{t}=0.50 \mathrm{~mm}$ and impact velocity $v_{i}=1.30 \mathrm{~m} / \mathrm{s}$. A slug of liquid that is disconnected from the rest of the drop is formed, due to a permanent cleaving, after the initial liquid penetration.

A more quantitative comparison between the numerical and experimental results, for the case with pore size $r_{t}=0.50 \mathrm{~mm}$ and impact velocity $v_{i}=1.30 \mathrm{~m} / \mathrm{s}$, is conducted in Figure 5 , where the position of the front meniscus scaled by the cylindrical tube (pore) radius $\mathrm{z} / \mathrm{r}_{\mathrm{t}}$ is plotted as a function of the dimensionless time $\tau=\operatorname{tr} \cos \theta / 2 \mu r_{t}$. 


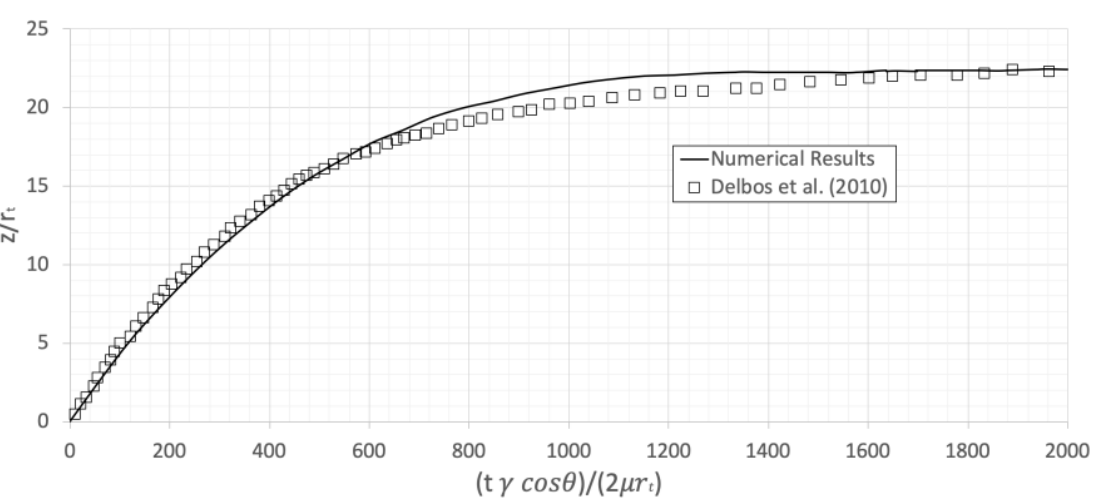

Figure 5. Quantitative comparison between the proposed numerical investigation results and the experimental data [33], for the case with tube radius $r_{t}=0.50 \mathrm{~mm}$ and impact velocity $v_{i}=1.30 \mathrm{~m} / \mathrm{s}$. Penetration depth $\mathrm{z}$ is scaled with the tube radius $r_{t}$ as a function of the dimensionless time $\tau$.

As it can be observed, the proposed numerical model successfully captures the transient evolution of the penetrating liquid front. All the above results indicate that the proposed numerical simulation framework can safely be applied for the proposed parametric numerical investigations of the present paper that consider quasi-sessile droplet depositions that are absorbed within single cylindrical pores of the same or similar pore sizes.

\section{Mesh Independence Study}

As mentioned previously, in order to adopt the utilised computational mesh characteristics, for the simulations presented in the paper, a mesh-independence study was conducted. In more detail, three different mesh densities were used for the case with tube radius $0.1 \mathrm{~mm}$ and impact velocity $v_{i} 0.15 \mathrm{~m} / \mathrm{s}$, consisting of $63,439,253,125$ and 1,012,500 cells. The corresponding uniform cell sizes for these three meshes are $4 \mu \mathrm{m}, 2 \mu \mathrm{m}$ and $1 \mu \mathrm{m}$, respectively. As it can be observed from Figure 6 , the coarse mesh predictions $(63,439$ cells) slightly deviate from the other two mesh cases. However, the results between the medium $(253,125$ cells) and fine meshes $(1,012,500)$, are almost identical. There are some small differences that can be considered negligible for the purposes of the present investigation. If the finer mesh was used instead of the medium one it would not add considerably more details, but it would significantly increase the computational time. Therefore, the medium mesh density can safely be selected as the mesh independent solution, for the purposes of the present parametric numerical investigation.

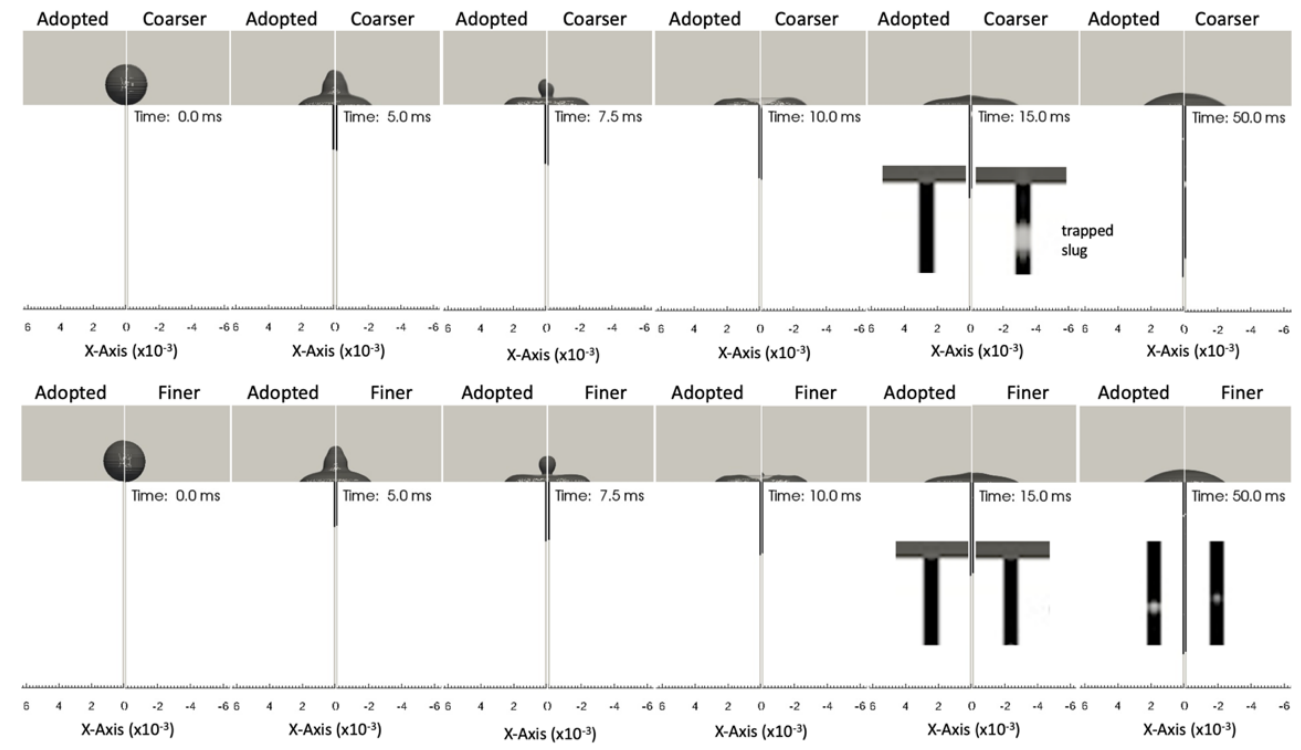

Figure 6. Mesh Independence Study: comparison of numerical predictions between the adopted mesh, a finer and a coarser mesh. 


\section{Parametric Numerical Simulations}

In the present section of the paper, the previously adopted numerical simulation set-up is further utilised in order to perform two different series of parametric numerical simulations. The main aim is to investigate the effects of pore size, liquid viscosity and initial droplet diameter for quasisessile droplets that are left to settle above a cylindrical pore with the bottom tip of the droplet in contact with the pore inlet (i.e. zero impact velocity). This is done in order to isolate, identify and quantify the absorption phenomenon without any initial momentum, as in the case of impacting droplets, in previous investigations. Furthermore, as reported in the literature typical exudate flow rates are really low, ranging from $0.2 \mathrm{~mL} / \mathrm{hr}$ up to $0.4 \mathrm{~mL} / \mathrm{hr}$ [45], which for typical pore sizes of wound dressings result in negligible values of velocity.

\subsection{Effects of pore size and liquid viscosity}

In order to investigate, identify and quantify the absorption characteristics of a liquid drop into a cylindrical pore, 48 in total, 2D-Axisymmetric simulations have been conducted with a quasisessile droplet of $2.5 \mathrm{~mm}$ in diameter, released in contact with the inlet of a pore, for cases with pore radius $0.05 \mathrm{~mm}, 0.10 \mathrm{~mm}, 0.20 \mathrm{~mm}, 0.30 \mathrm{~mm}, 0.40 \mathrm{~mm}$ and $0.50 \mathrm{~mm}$ and kinematic viscosity 1.0 $\times 10^{-6} \mathrm{~m}^{2} / \mathrm{s}, 1.2 \times 10^{-6} \mathrm{~m}^{2} / \mathrm{s}, 1.5 \times 10^{-6} \mathrm{~m}^{2} / \mathrm{s}, 1.8 \times 10^{-6} \mathrm{~m}^{2} / \mathrm{s}, 2 \times 10^{-6} \mathrm{~m}^{2} / \mathrm{s}, 4 \times 10^{-6} \mathrm{~m}^{2} / \mathrm{s}, 6 \times 10^{-6} \mathrm{~m}^{2} / \mathrm{s}$ and $8 \times 10^{-6} \mathrm{~m}^{2} / \mathrm{s}$. Details of the computational domain and the boundary conditions have already been illustrated in Figure 1, where the validation runs were analysed and presented. The overall cases simulated, and their main characteristics are summarised in Table I. All simulations were run for a total flow time of $50 \mathrm{~ms}$ or up to the time instance that the absorbed liquid front has reached the bottom end of the computational domain. However, in some cases, the droplet evolution comes to an equilibrium well before the $50 \mathrm{~ms}$. A generalised schematic of the initial condition for these simulations is shown in Figure 7. It should be mentioned here, that in all of the simulated cases, apart from the liquid kinematic viscosity that constitutes one of the varied parameters, the rest of the fluid properties for the liquid phase and the gaseous phase remain unchanged and equal to the property values that were used for the validation runs.

Table I. Main characteristics of simulated cases.

\begin{tabular}{|c|c|c|c|c|c|c|c|c|c|c|c|}
\hline Case & $\begin{array}{c}\text { D }_{\text {pore }} \\
{[\mathrm{mm}]}\end{array}$ & $\begin{array}{c}\boldsymbol{v} \\
{\left[\mathrm{m}^{2} / \mathrm{s}\right]}\end{array}$ & $\begin{array}{l}\text { D droplet } \\
\text { [mm] }\end{array}$ & Case & $\begin{array}{c}\text { D }_{\text {pore }} \\
{[\mathrm{mm}]}\end{array}$ & $\begin{array}{c}\boldsymbol{v} \\
{\left[\mathrm{m}^{2} / \mathrm{s}\right]}\end{array}$ & $\begin{array}{l}\text { Ddroplet }_{\text {[re }} \\
\text { [mm] }\end{array}$ & Case & $\begin{array}{c}D_{\text {pore }} \\
{[\mathrm{mm}]}\end{array}$ & $\begin{array}{c}\boldsymbol{v} \\
{\left[\mathrm{m}^{2} / \mathrm{s}\right]}\end{array}$ & $\begin{array}{c}\text { Ddroplet } \\
{[\text { [mm] }}\end{array}$ \\
\hline C1-1 & \multirow[t]{8}{*}{0.1} & $1.0 \times 10^{-6}$ & \multirow{16}{*}{2.5} & C3-1 & \multirow{8}{*}{0.4} & $1.0 \times 10^{-6}$ & \multirow{16}{*}{2.5} & C5-1 & \multirow{8}{*}{0.8} & $1.0 \times 10^{-6}$ & \multirow{16}{*}{2.5} \\
\hline C1-2 & & $1.2 \times 10^{-6}$ & & C3-2 & & $1.2 \times 10^{-6}$ & & C5-2 & & $1.2 \times 10^{-6}$ & \\
\hline C1-3 & & $1.5 \times 10^{-6}$ & & C3-3 & & $1.5 \times 10^{-6}$ & & C5-3 & & $1.5 \times 10^{-6}$ & \\
\hline C1-4 & & $1.8 \times 10^{-6}$ & & C3-4 & & $1.8 \times 10^{-6}$ & & C5-4 & & $1.8 \times 10^{-6}$ & \\
\hline C1-5 & & $2.0 \times 10^{-6}$ & & C3-5 & & $2.0 \times 10^{-6}$ & & C5-5 & & $2.0 \times 10^{-6}$ & \\
\hline C1-6 & & $4.0 \times 10^{-6}$ & & C3-6 & & $4.0 \times 10^{-6}$ & & C5-6 & & $4.0 \times 10^{-6}$ & \\
\hline C1-7 & & $6.0 \times 10^{-6}$ & & C3-7 & & $6.0 \times 10^{-6}$ & & C5-7 & & $6.0 \times 10^{-6}$ & \\
\hline C1-8 & & $8.0 \times 10^{-6}$ & & C3-8 & & $8.0 \times 10^{-6}$ & & C5-8 & & $8.0 \times 10^{-6}$ & \\
\hline C2-1 & \multirow[t]{8}{*}{0.2} & $1.0 \times 10^{-6}$ & & C4-1 & \multirow{8}{*}{0.6} & $1.0 \times 10^{-6}$ & & C6-1 & \multirow{8}{*}{1.0} & 1. $0 \times 10^{-6}$ & \\
\hline C2-2 & & $1.2 \times 10^{-6}$ & & C4-2 & & $1.2 \times 10^{-6}$ & & C6-2 & & $1.2 \times 10^{-6}$ & \\
\hline $\mathrm{C} 2-3$ & & $1.5 \times 10^{-6}$ & & C4-3 & & $1.5 \times 10^{-6}$ & & C6-3 & & $1.5 \times 10^{-6}$ & \\
\hline C2-4 & & $1.8 \times 10^{-6}$ & & C4-4 & & $1.8 \times 10^{-6}$ & & C6-4 & & $1.8 \times 10^{-6}$ & \\
\hline C2-5 & & $2.0 \times 10^{-6}$ & & C4-5 & & $2.0 \times 10^{-6}$ & & C6-5 & & $2.0 \times 10^{-6}$ & \\
\hline C2-6 & & $4.0 \times 10^{-6}$ & & C4-6 & & $4.0 \times 10^{-6}$ & & C6-6 & & $4.0 \times 10^{-6}$ & \\
\hline C2-7 & & $6.0 \times 10^{-6}$ & & C4-7 & & $6.0 \times 10^{-6}$ & & C6-7 & & $6.0 \times 10^{-6}$ & \\
\hline $\mathrm{C2}-8$ & & $8.0 \times 10^{-6}$ & & C4-8 & & $8.0 \times 10^{-6}$ & & C6-8 & & $8.0 \times 10^{-6}$ & \\
\hline
\end{tabular}




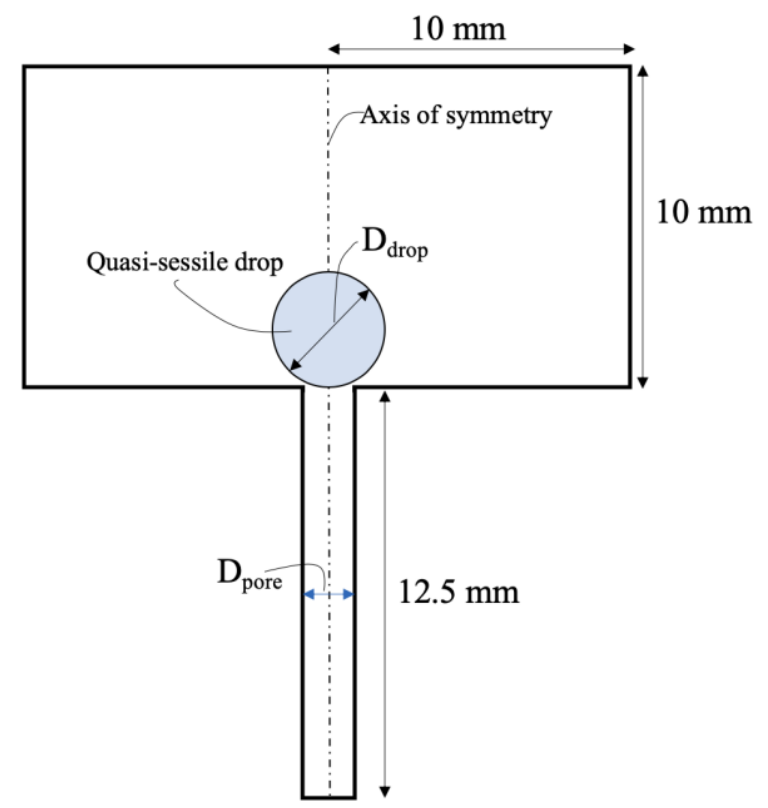

Figure 7. Generalised schematic of initial condition for the considered numerical simulations (not to scale)

Figures 8, 9, 12, 13, 14 and 17, illustrate the variation of the liquid volume fraction within the cylindrical pore $\left(\frac{V_{\text {liquid }}}{V_{\text {pore }}}\right)$ with respect to a dimensionless time $\tau$, varying the liquid viscosity for different pore sizes. It should be mentioned that the graph on the left, groups the points with respect to each viscosity value (different series for each viscosity), while the graph on the right, uses a general scatter depending on the observed flow regime, in order for best-fit lines to be obtained. Focusing first on the small pore sizes among the ones examined, in Figures $8\left(r_{\text {pore }}=0.05 \mathrm{~mm}\right)$ and $9\left(r_{\text {pore }}=0.1\right.$ $\mathrm{mm}$ ), it is evident that the points of liquid volume fraction within the pore versus dimensionless time, despite the variation of the liquid viscosity, are all collapsing on a single curve with a relatively low scatter, for each particular pore value. The best fit line in each case, can be described by a different power law with a very good fit. In more detail, as it can be seen from the graphs, the $\mathrm{R}^{2}$ value is 0.9775 and 0.9736 for the cases with pore radius $0.05 \mathrm{~mm}$ and $0.1 \mathrm{~mm}$, respectively.
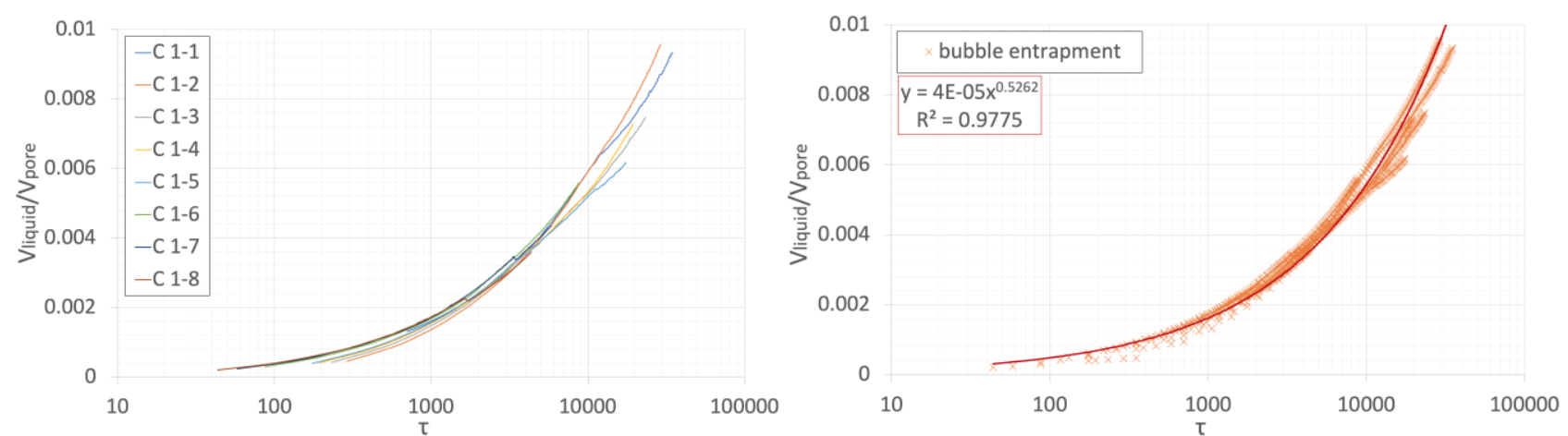

Figure 8. The liquid volume fraction inside the capillary against dimensionless time, varying the liquid viscosity for pore radius of $0.05 \mathrm{~mm}$ (Please refer to Table 1 for the cases' characteristics). Cases grouped by viscosity (left); cases grouped by observed flow regime (right). 

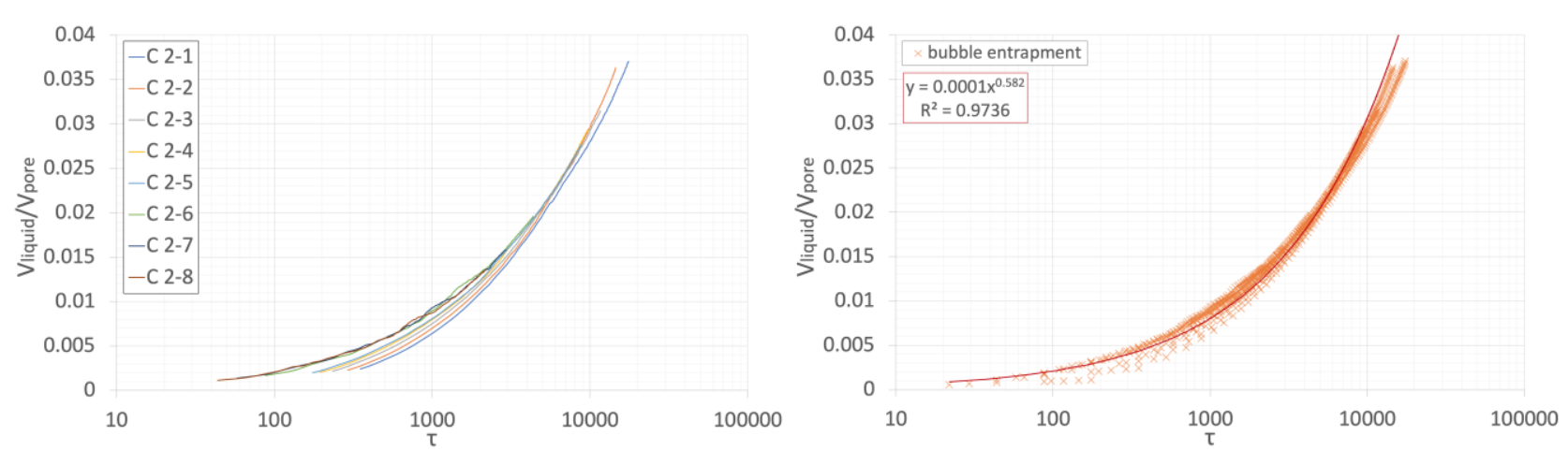

Figure 9. The liquid volume fraction inside the capillary against dimensionless time, varying the liquid viscosity for pore radius of $0.1 \mathrm{~mm}$ (Please refer to Table 1 for the cases' characteristics). Cases grouped by viscosity (left); cases grouped by observed flow regime (right).

Furthermore, examining macroscopic, flow visualisation results for all these 16 cases (Figures 8 and 9), it is evident from Figure $10\left(r_{\text {pore }}=0.05 \mathrm{~mm}\right)$ and Figure $11\left(r_{\text {pore }}=0.1 \mathrm{~mm}\right)$ that for the two smaller pore sizes from the ones examined, the evolution of the liquid penetration within the cylindrical pore follows a single flow regime, characterised by bubble entrapment, despite the variation of the liquid viscosity. In more detail, the liquid droplet penetrates the cylindrical pore, filling it up progressively, while at some time instance in each case, a pinch-off event that occurs at the liquid column directly above the pore, entraps a small air bubble, that then disintegrates into smaller bubbles as it is absorbed within the pore. These smaller bubbles are then penetrating further into the pore. At this point, it should be mentioned that the light grey spots that appear in the main drop evolution above the pore at some time instances in Figures 10, 11, 15, 16, 18-20 are lighting/shadowing effects from the postprocessor and not air bubbles.

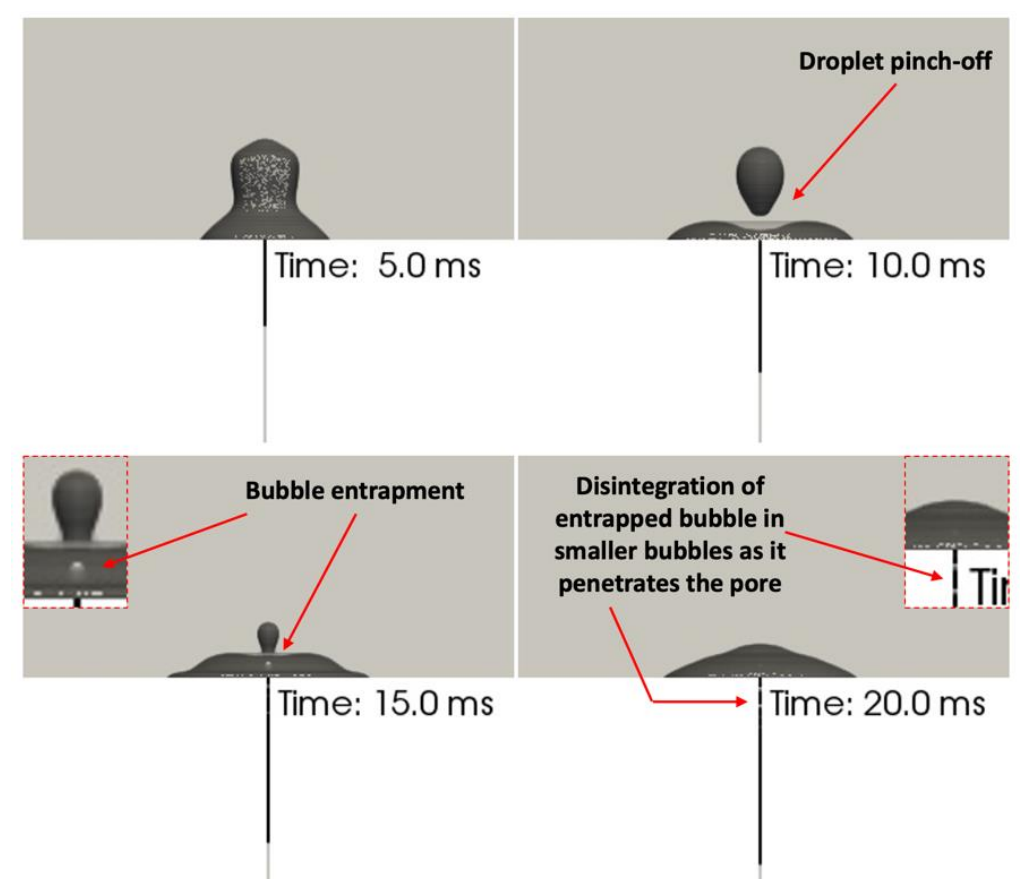

Figure 10. Indicative droplet evolution for case with pore radius, $\mathrm{r}_{\mathrm{pore}}=0.05 \mathrm{~mm}$, and liquid kinematic viscosity, $v=1 \times 10^{-6} \mathrm{~m}^{2} / \mathrm{s}$ (Case $1-1$ from Table 1$)$. 


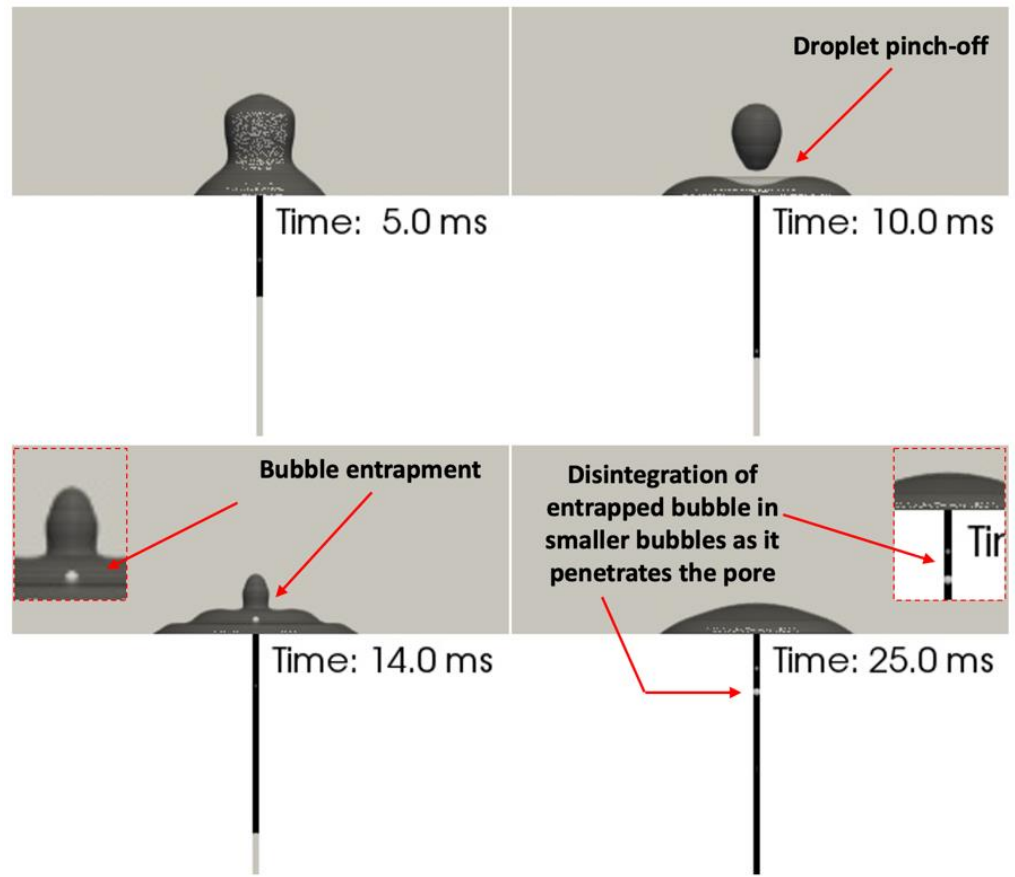

Figure 11. Indicative droplet evolution for case with pore radius, $r_{\mathrm{pore}}=0.1 \mathrm{~mm}$, and liquid kinematic viscosity, $v=1 \times 10^{-6} \mathrm{~m}^{2} / \mathrm{s}$ (Case 2-1 from Table 1$)$.

Focusing now on medium pore sizes among the ones examined, in Figure $11\left(\mathrm{r}_{\text {pore }}=0.2 \mathrm{~mm}\right)$, Figure $12\left(r_{\text {pore }}=0.3 \mathrm{~mm}\right)$ and Figure $13\left(r_{\text {pore }}=0.4 \mathrm{~mm}\right)$, it is evident that the points of liquid volume fraction within the pore versus dimensionless time are, depending on the viscosity value, collapsing on two different curves, for each pore size, again with a relatively low scatter. The best fit lines for cases of pore radius of $r_{\text {pore }}=0.2$ and $r_{\text {pore }}=0.3$, can be described by two different power laws, in each case, with a good fit in general that progressively weakens with the increase of the pore radius $\left(\mathrm{R}^{2}\right.$ values are 0.9538 and 0.9663 for the case with pore radius $0.2 \mathrm{~mm}$, and 0.9181 and 0.9203 for the case with pore radius $0.3 \mathrm{~mm}$ ). For pore radius $\mathrm{r}_{\text {pore }}=0.4$, however, the best fit lines for the corresponding two curves are best described by a second order polynomial and not by a power law. The fit in this case is quite good $\left(\mathrm{R}^{2}\right.$ values are 0.9554 and 0.9864$)$. It should be mentioned that in all these three pore size cases, the black best fit line corresponds to high viscosity values $\left(4 \times 10^{-6} \mathrm{~m}^{2} / \mathrm{s} \leq v \leq 8 \times 10^{-6} \mathrm{~m}^{2} / \mathrm{s}\right)$ and the red one corresponds to low viscosity values $\left(1 \times 10^{-6} \mathrm{~m}^{2} / \mathrm{s} \leq v \leq 2 \times 10^{-6} \mathrm{~m}^{2} / \mathrm{s}\right)$. This indicates that irrespectively of the pore radius for the proposed range of radii $\left(0.2 \mathrm{~mm} \leq \mathrm{r}_{\text {pore }} \leq 0.4 \mathrm{~mm}\right)$ there seems to be a threshold viscosity value that changes the liquid absorption rate.
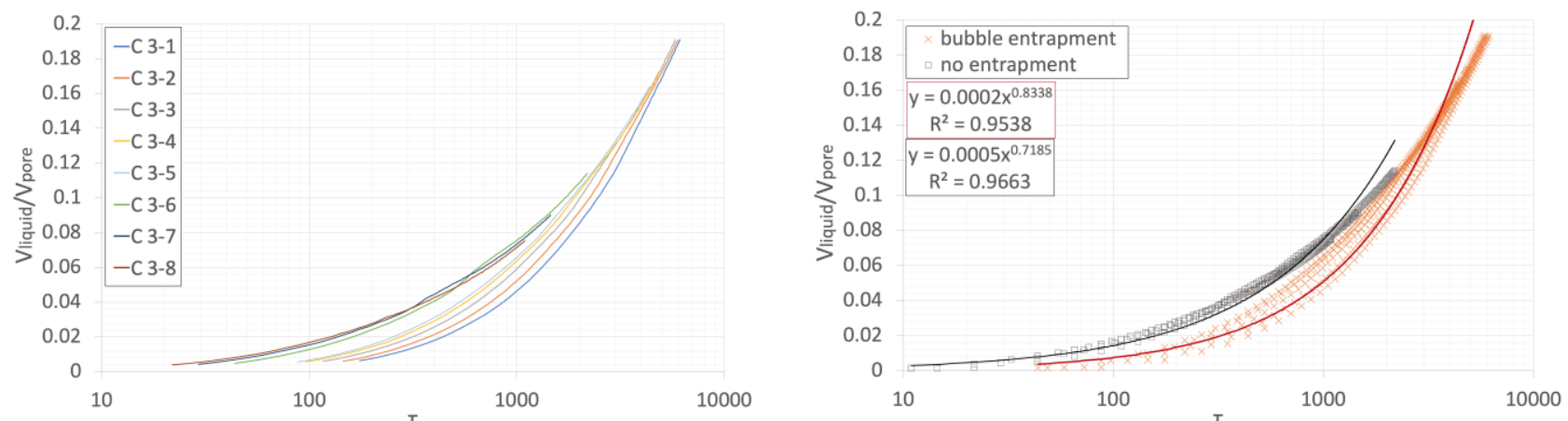

Figure 12. The liquid volume fraction inside the capillary against dimensionless time, varying the liquid viscosity for pore radius of $0.2 \mathrm{~mm}$ (Please refer to Table 1 for the cases' characteristics). Cases grouped by viscosity (left); cases grouped by observed flow regime (right). 

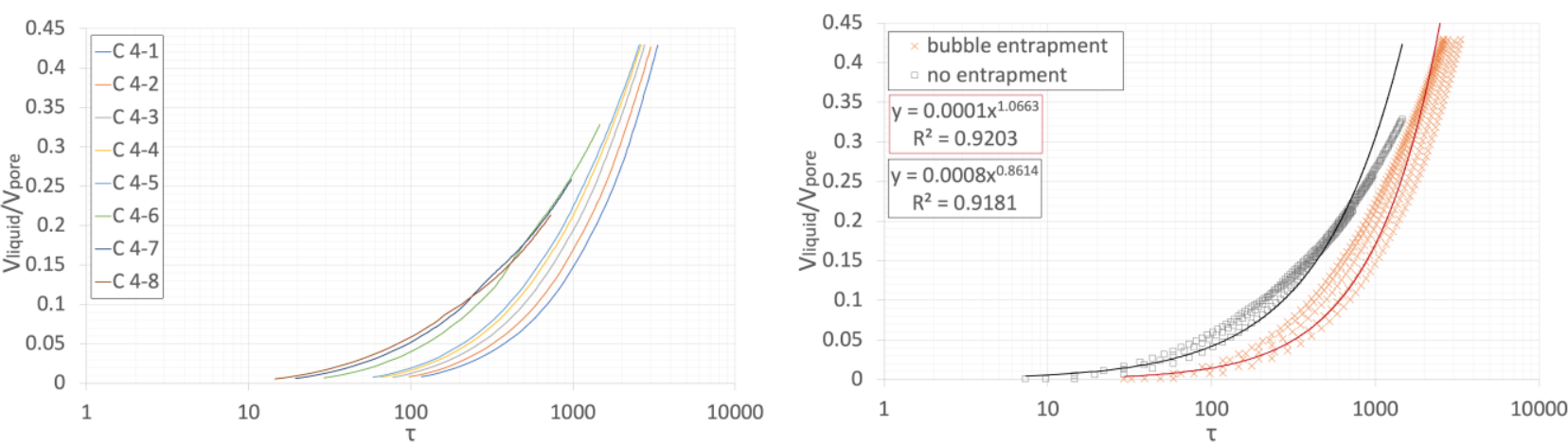

Figure 13. The liquid volume fraction inside the capillary against dimensionless time, varying the liquid viscosity for pore radius of $0.3 \mathrm{~mm}$ (Please refer to Table 1 for the cases' characteristics). Cases grouped by viscosity (left); cases grouped by observed flow regime (right).
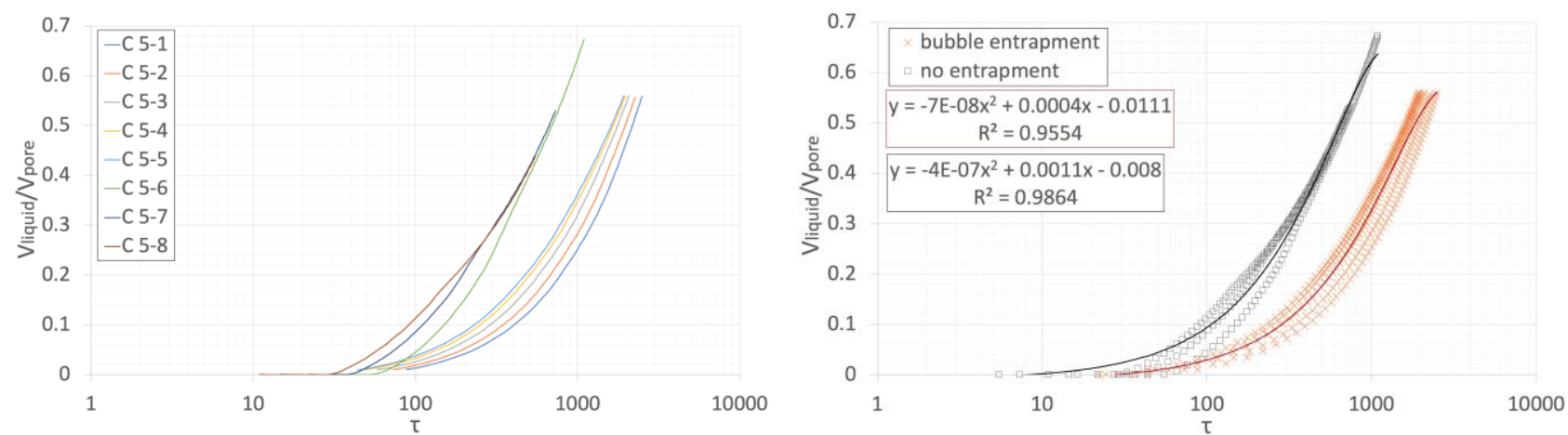

Figure 14. The liquid volume fraction inside the capillary against dimensionless time, varying the liquid viscosity for pore radius of $0.4 \mathrm{~mm}$ (Please refer to Table 1 for the cases' characteristics). Cases grouped by viscosity (left); cases grouped by observed flow regime (right).

Examining macroscopic flow visualisation results for all these 24 cases (Figures 12, 13 and 14), it is evident from the indicatively selected cases illustrated in Figure 15 and Figure 16 that for each pore size, depending on the aforementioned viscosity ranges, two distinct droplet evolution regimes are present. In more detail, the low viscosity range related regime is the same as the one described previously in Figures 10 and 11, which is indicatively shown here in Figure 15, for a particular viscosity value and pore size (Case 3-1 from Table 1). However, for the high viscosity range related regime, not any droplet pinch-off is observed for all considered cases and therefore the liquid that is absorbed, penetrates progressively within the pore without any air bubble entrapment. This regime is indicatively shown for a particular viscosity value and pore size (Case 3-8 from Table 1), in Figure 16. It should be mentioned that for the case illustrated in Figure 16, the droplet evolution comes to a quasi-equilibrium state at $\mathrm{t}=30 \mathrm{~ms}$, with negligible change up to $50 \mathrm{~ms}$. 


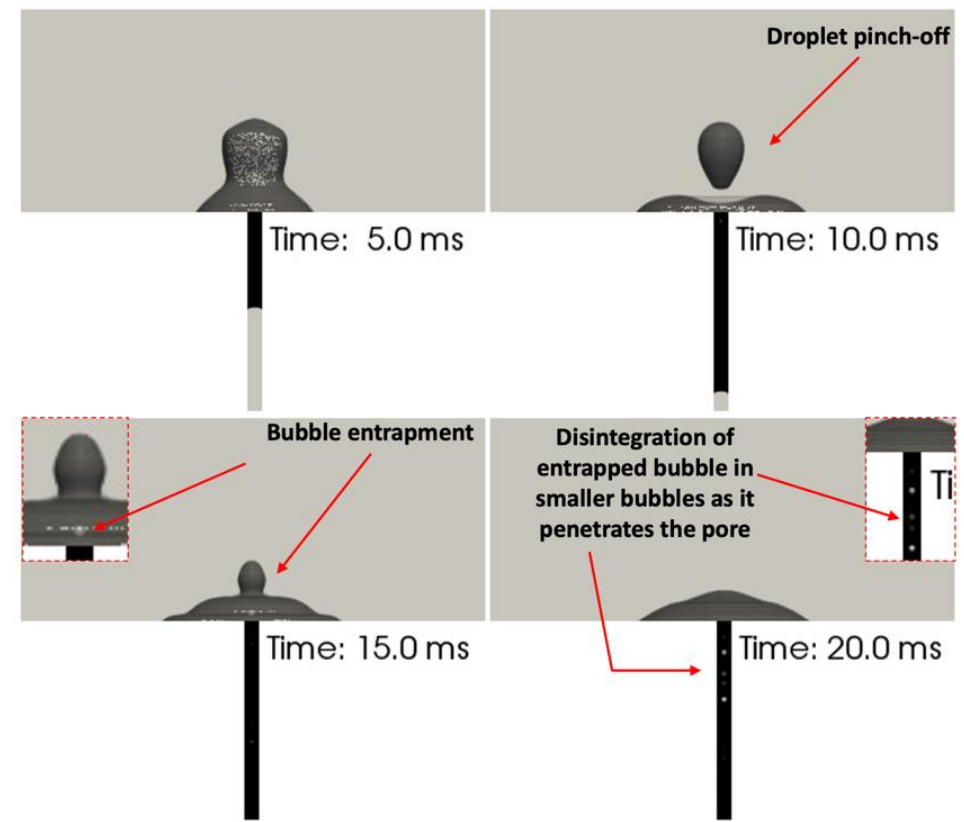

Figure 15. Indicative droplet evolution for case with pore radius, $r_{\text {pore }}=0.2 \mathrm{~mm}$, and liquid kinematic viscosity, $v=1 \times 10^{-6} \mathrm{~m}^{2} / \mathrm{s}$ (Case $3-1$ from Table 1$)$.
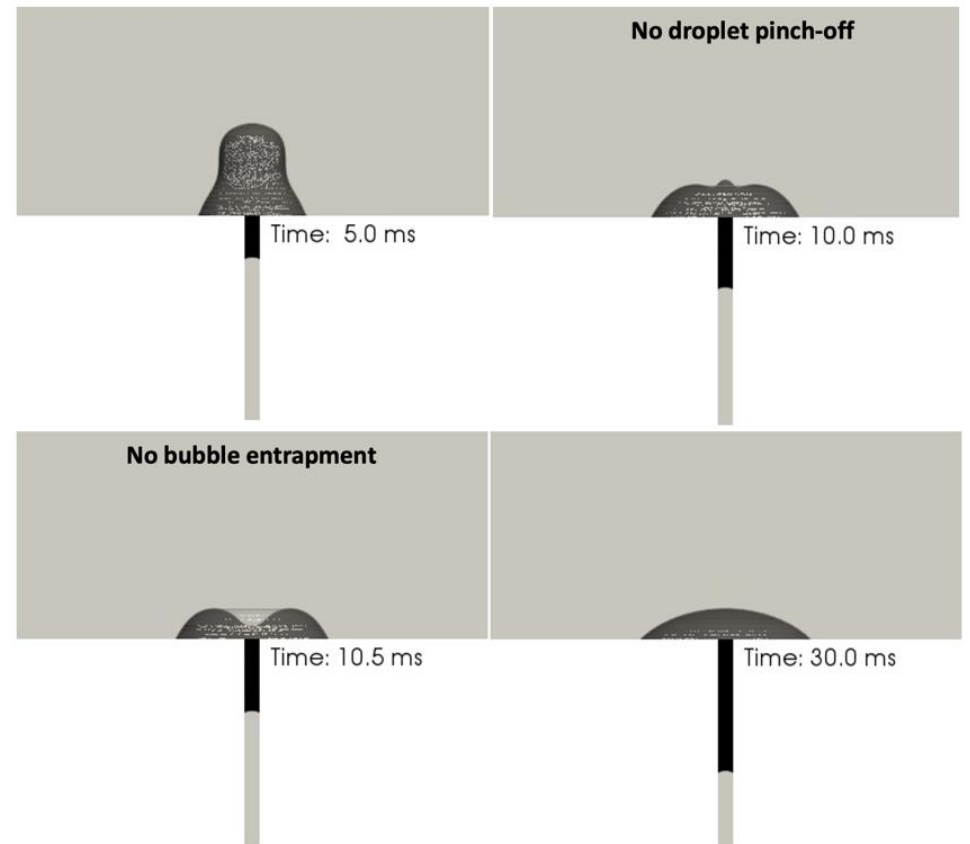

Figure 16. Indicative droplet evolution for case with pore radius, $r_{\text {pore }}=0.2 \mathrm{~mm}$, and liquid kinematic viscosity, $v=8 \times 10^{-6} \mathrm{~m}^{2} / \mathrm{s}$ (Case $3-8$ from Table 1$)$.

Finally, focusing on the highest pore size among the ones examined, in Figure 17 ( $r_{\text {pore }}=0.5$ $\mathrm{mm}$ ), it is evident that the points of liquid volume fraction within the pore versus dimensionless time are, depending on the viscosity value, collapsing on three different curves, for the particular pore size, again with a relatively low scatter. The best-fit lines can be described by three different second order polynomial relationships. In more detail, as it can be seen from the graphs in Figure 17, the corresponding $\mathrm{R}^{2}$ values are 0.9707 for the blue best-fit line that corresponds to low viscosities $\left(1 \times 10^{-6} \mathrm{~m}^{2} / \mathrm{s} \leq v \leq 1.2 \times 10^{-6} \mathrm{~m}^{2} / \mathrm{s}\right), 0.8348$ for the green best-fit line that corresponds to medium viscosities $\left(1.5 \times 10^{-6} \mathrm{~m}^{2} / \mathrm{s} \leq v \leq 4 \times 10^{-6} \mathrm{~m}^{2} / \mathrm{s}\right)$ and 0.9939 for the black best-fit line that corresponds to high viscosities $\left(6 \times 10^{-6} \mathrm{~m}^{2} / \mathrm{s} \leq v \leq 8 \times 10^{-6} \mathrm{~m}^{2} / \mathrm{s}\right)$, respectively. This indicates that for relatively large pore radii $\left(\mathrm{r}_{\text {pore }} \geq 0.5 \mathrm{~mm}\right.$ ), there seems to be two different threshold viscosity values that change the liquid absorption rate. 

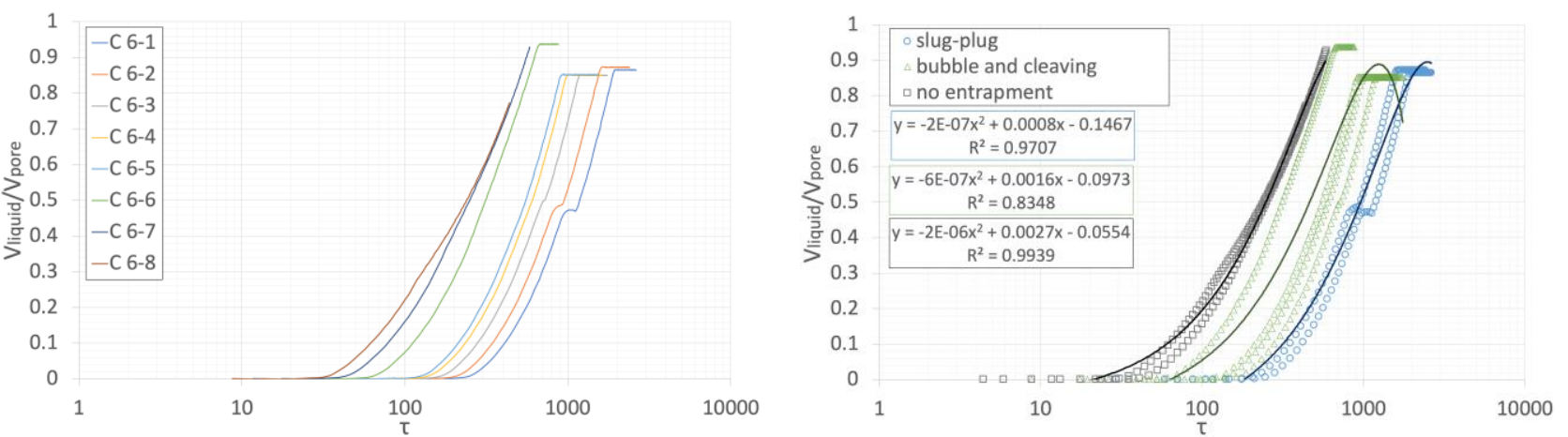

Figure 17. The liquid volume fraction inside the capillary against dimensionless time, varying the liquid viscosity for pore radius of $0.5 \mathrm{~mm}$ (Please refer to Table 1 for the cases' characteristics). Cases grouped by viscosity (left); cases grouped by observed flow regime (right).

Examining macroscopic flow visualisation results for these last eight cases (Figure 17), it is evident from the indicatively selected cases illustrated in Figures 18, 19 and 20 that for the particular pore size, depending on the aforementioned viscosity ranges, three distinct droplet evolution regimes are present. In more detail, the low viscosity range related regime is depicted indicatively in Figure 18 (Case 6-1 from Table 1) where, as it can be observed, the liquid droplet penetrates the cylindrical pore, filling it up progressively, while at some time instance, a pinch-off event that occurs in combination with a subsequent cleaving event, entraps an elongated air plug that progressively penetrates further into the pore. At the end, a second cleaving event is also observed. For the medium viscosity range related regime, the observed droplet evolution is the same as the regime presented and described in Figure 10 but accompanied by a cleaving event at the end. This is indicatively shown in Figure 19 for a particular viscosity value (Case 6-4 from Table 1). Finally, for the high viscosity range related regime, the observed droplet evolution is the same as the regime presented in Figure 16, where no bubble entrapment occurs. This is illustrated indicatively for a particular viscosity value also for the considered pore radius $\left(\mathrm{r}_{\text {pore }}=0.5 \mathrm{~mm}\right.$ ) in Figure 20 (Case 6-8 from Table 1). It should be mentioned that for the case illustrated in Figure 20 the droplet evolution comes to a quasi-equilibrium state at $\mathrm{t}=30 \mathrm{~ms}$, with negligible change up to $50 \mathrm{~ms}$.

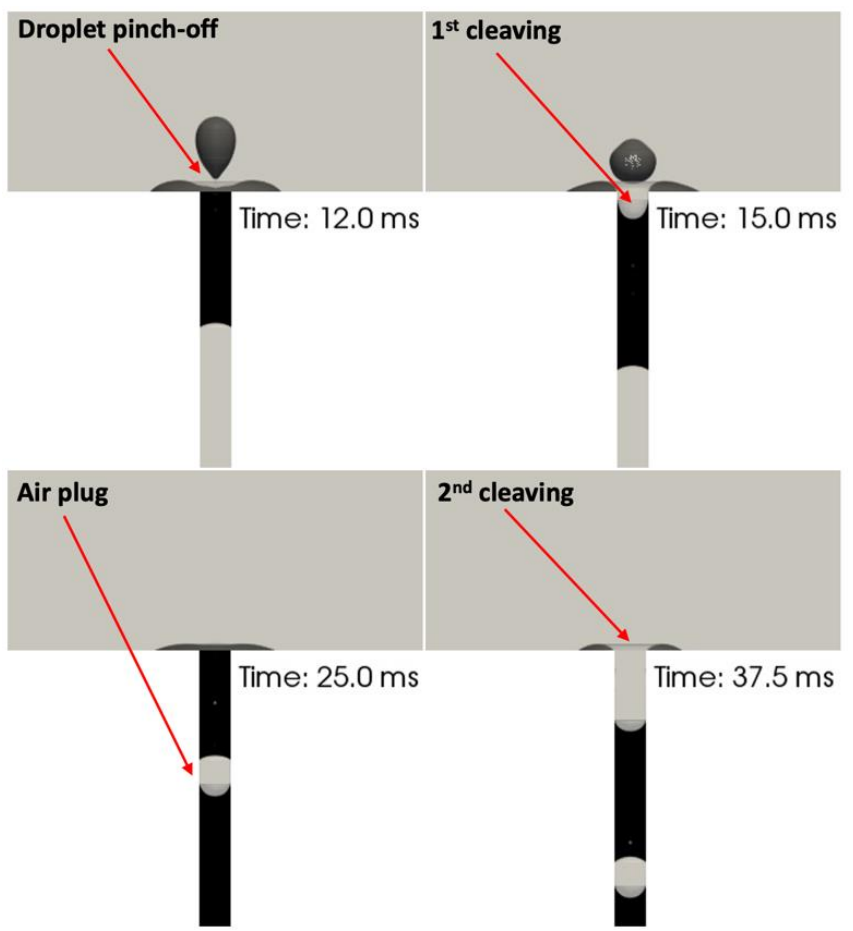

Figure 18. Indicative droplet evolution for case with pore radius, $r_{\mathrm{pore}}=0.5 \mathrm{~mm}$, and liquid kinematic viscosity, $v=1 \times 10^{-6} \mathrm{~m}^{2} / \mathrm{s}$ (Case $6-1$ from Table 1 ). 


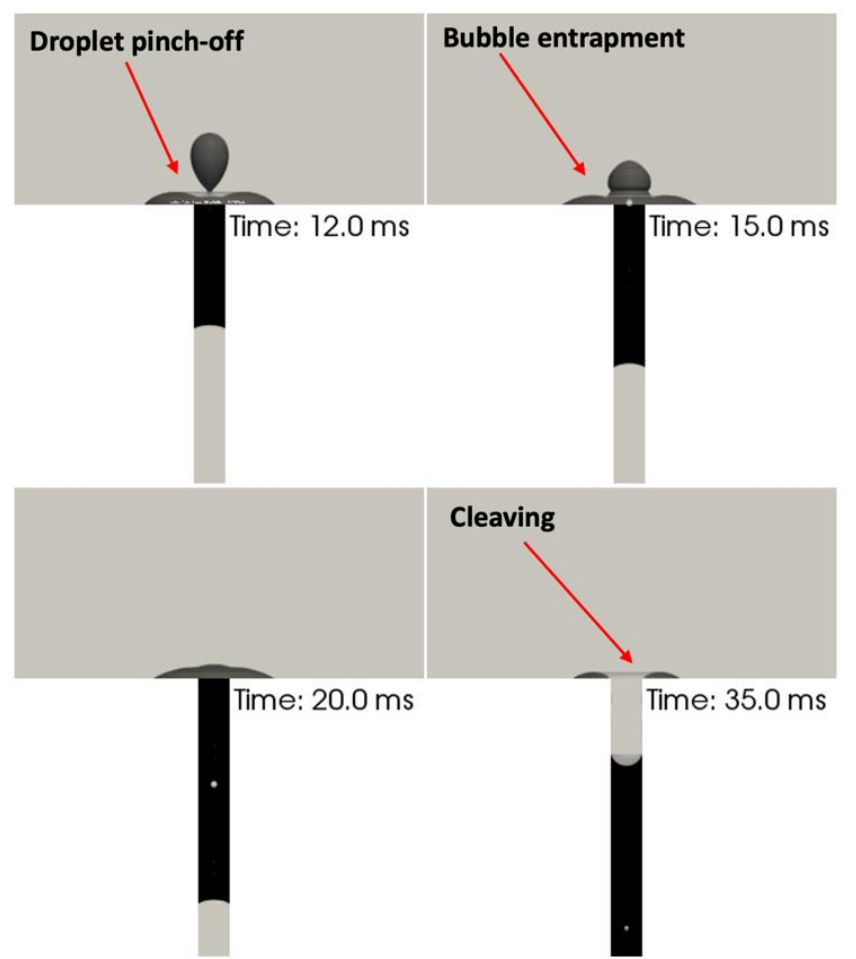

Figure 19. Indicative droplet evolution for case with pore radius, $r_{\mathrm{pore}}=0.5 \mathrm{~mm}$, and liquid kinematic viscosity, $v=1.8 \times 10^{-6} \mathrm{~m}^{2} / \mathrm{s}$ (Case $6-4$ from Table 1$)$.
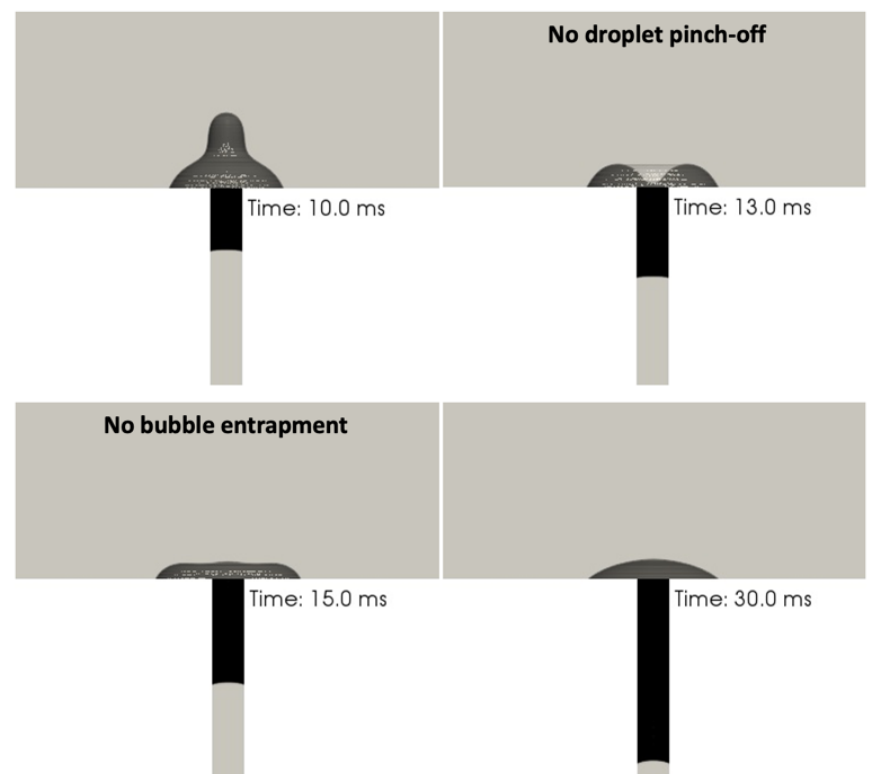

Figure 20. Indicative droplet evolution for case with pore radius, $r_{\mathrm{pore}}=0.5 \mathrm{~mm}$, and liquid kinematic viscosity, $v=8 \times 10^{-6} \mathrm{~m}^{2} / \mathrm{s}$ (Case $6-8$ from Table 1$)$.

From all the above, it can be concluded that among all of the 48 examined quasi-sessile drop cases, four distinct droplet flow regimes have been identified that are directly linked with the distinct droplet absorption rates that were identified from the simulation predictions,

- bubble entrapment;

- bubble entrapment and cleaving;

- slug-plug flow and cleaving and;

- no entrapment.

For small pore sizes, a single flow regime is identified for all of the examined liquid viscosities. For medium pore sizes, two distinct flow regimes are revealed, one for low and one for 
high liquid viscosities. Finally, for large pores three distinct flow regimes occur, for low, medium and high viscosity value ranges. In each case relationships have been derived, using best-fit lines, that can predict the absorption rates for particular values of pore size and liquid viscosity. It is characteristic that for small pore sizes $(0.05 \mathrm{~mm}$ and $0.1 \mathrm{~mm}$, in Figures 8 and 9$)$, the resulting relationship from the best fit lines resembles the classic Washburn's equation for capillary flows [46], since the liquid volume fraction is approximately proportional to the square root of the dimensionless time. However, for higher pore sizes $(0.2 \mathrm{~mm}$ and $0.3 \mathrm{~mm}$, in Figures 12 and 13) the data deviate from the proposed proportionality. This indicates that the liquid penetration behaviour that is described by Washburn's equation is valid for relatively low pore sizes.

\subsection{Effect of droplet size}

In order to also investigate the effect of the drop size on the droplet absorption characteristics, 18 additional simulations have been performed for droplet diameter $1.5 \mathrm{~mm}$ and $3.5 \mathrm{~mm}$ and for the cases with pore radius $0.05 \mathrm{~mm}, 0.3 \mathrm{~mm}$ and $0.5 \mathrm{~mm}$ and with kinematic viscosities $10^{-6} \mathrm{~m}^{2} / \mathrm{s}, 4 \times 10^{-6}$ $\mathrm{m}^{2} / \mathrm{s}$ and $8 \times 10^{-6} \mathrm{~m}^{2} / \mathrm{s}$. The results from this parametric analysis investigation are depicted in Figure 21 , where the 27 in total cases are examined (including the corresponding cases for droplet diameter of $2.5 \mathrm{~mm}$, from the previous parametric analysis in sub-section 5.1). As it can be observed, for the cases with small pore diameter $\left(\mathrm{r}_{\text {pore }}=0.05 \mathrm{~mm}\right.$ ) and high values of viscosity (yellow curves), the droplet size does not play a significant role in the droplet absorption characteristics. However, as the viscosity values decrease (orange and blue curves) the effect of the initial droplet diameter progressively becomes more significant, especially for the latter stages of the droplet evolution. For medium pore sizes $\left(\mathrm{r}_{\mathrm{pore}}=0.3 \mathrm{~mm}\right)$, as it can be observed the variation of the droplet diameter has an insignificant effect in the droplet absorption rate. It should be highlighted here that the horizontal segments of the curves, for the cases of small droplet diameter $(\mathrm{D}=1.5 \mathrm{~mm})$ and for low and medium viscosities (yellow and orange dashed line curves), correspond to the time periods that almost the entire volume of the initial droplet has been completely absorbed within the pore accompanied by a permanent cleaving. Therefore, during this stage, the volume fraction of the liquid within the pore remains constant as the further absorption of the droplet has been interrupted by the proposed cleaving event. Before these time periods, the droplet absorption rate is almost the same for all three values of the initial droplet diameter. Finally, for bigger pore sizes $\left(r_{\text {pore }}=0.5 \mathrm{~mm}\right)$, it is characteristic that for the smallest droplet diameter the viscosity does not play any role at all since the considered droplet in all of the three examined viscosity cases, shows almost the same absorption rate and in all three cases it is completely absorbed within the pore at similar time instances. For medium and high viscosities, taking into consideration the two highest droplet diameters, it can be seen that the droplet diameter variation does not affect the absorption rate. The horizontal segment in the case with $\mathrm{D}=2.5 \mathrm{~mm}$ and medium viscosity (orange curve), that starts at point $\mathrm{g}$, corresponds to the time period after a final permanent cleaving event that interrupts the further absorption of the droplet within the pore. Finally, for low viscosity and taking into consideration the two highest droplet diameters, the initial stages of the droplet absorption almost coincide, but after a particular time instance a droplet break-up/pinch-off event occurs (points a and $\mathrm{c}$ in the graph) that is accompanied by a subsequent merging of the initially pinched-off droplet (points $b$ and d) back to the mother droplet (12ms and $16 \mathrm{~ms}$ for the $2.5 \mathrm{~mm}$ droplet, and $17 \mathrm{~ms}$ and $22.5 \mathrm{~ms}$ for the $3.5 \mathrm{~mm}$ droplet) occurs, encapsulating an air plug within the liquid flow. Between these two subsequent events, the liquid absorption within the pore pauses and resumes. As it can be seen, this pause happens at different stages for the two different initial droplet diameters, creating the observed deviations in the resulting curves. However, it is characteristic that the resumed absorption stages in each case still have similar rates that are almost the same with the initial absorption rate. Finally, the horizonal segments that begin at points $f$ and e, correspond to the final permanent cleaving stages, in each case. 

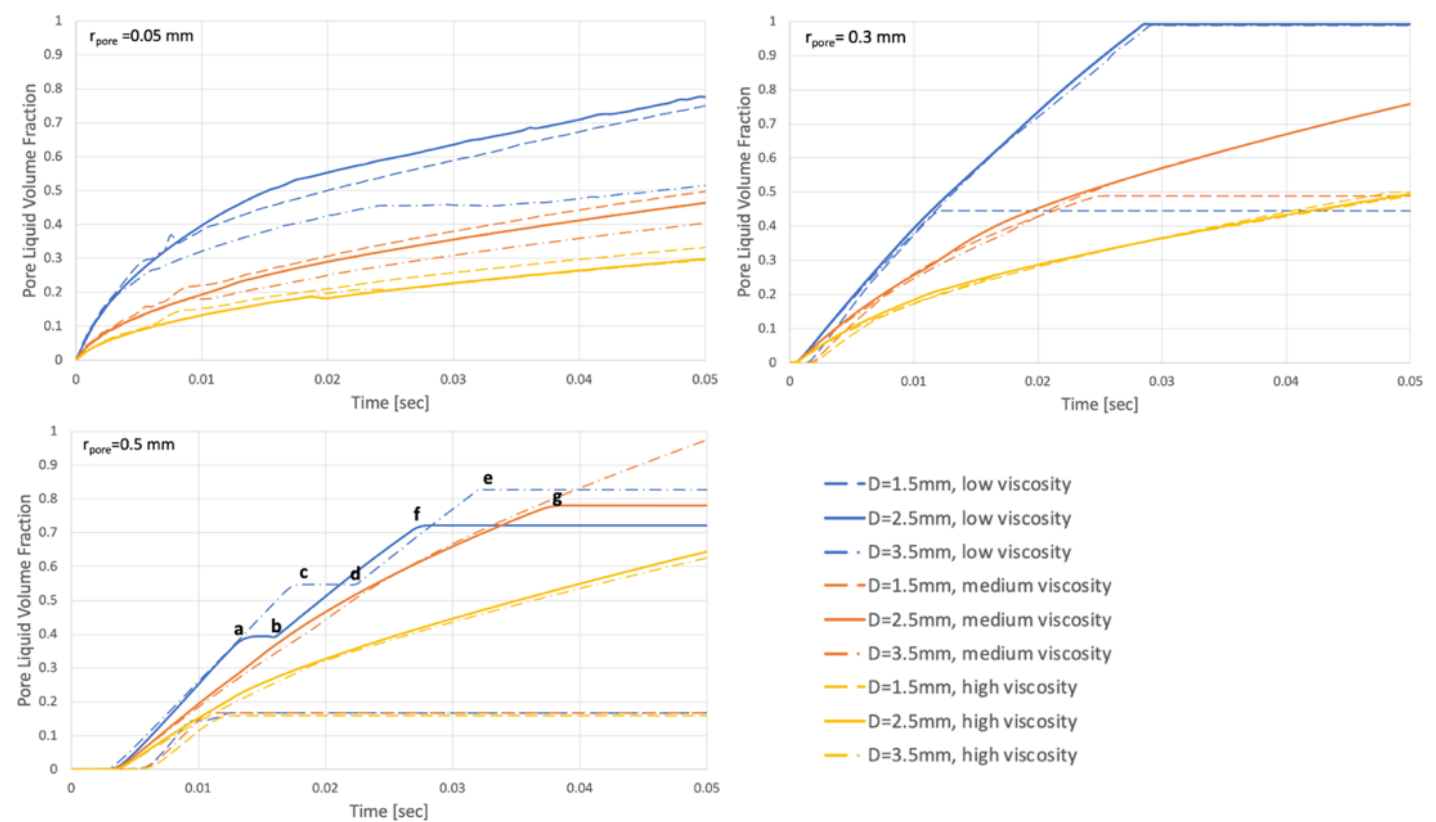

Figure 21. The liquid volume fraction inside the capillary against time, varying the liquid viscosity and the droplet diameter for three different pore sizes.

Summarising, in general, the initial droplet diameter does not seem to significantly influence the liquid absorption rate, apart from cases of small pore size and low viscosity liquids. Finally, it should also be mentioned that the previously identified droplet absorption regimes for the initial droplet diameter of $\mathrm{D}=2.5 \mathrm{~mm}$ (Sub-section 5.1), depending on the pore size and liquid viscosity, are also respected by the smaller and larger droplet diameter cases that were examined in the present subsection (Sub-section 5.2).

\section{Mapping of Flow Regimes}

As mentioned in the previous section, among all of the simulations presented and discussed in the present paper four distinct flow regimes have been identified that depend on the pore size and the liquid viscosity (Fig. 22); a) bubble entrapment, b) bubble entrapment and cleaving, c) slug-plug flow and cleaving and, d) no entrapment. It is reasonable to assume that the most desired regimes for wound healing purposes, in order to maximise the would exudate absorption into the pores of the wound dressing, and hence, keep it saturated (as much as possible), are the "no entrapment" regime as well as the "bubble entrapment regime", where only small air bubbles are entrapped into the penetrating liquid. On the contrary, the other two regimes ("bubble entrapment and cleaving" and "slug-plug flow and cleaving") should not be desired, due to the fact that quite large quantity of air penetrates into the pores and therefore the wound dressing might not be saturated enough, in order to keep the wound moist. Hence, ideally, there should be critical limits in characteristic dimensionless parameters that define the desired flow conditions.

Following a dimensional analysis, a flow map with the aforementioned regimes has been constructed, using the data from all 66 simulations, and calculating the corresponding Eötvos numbers (Eo) and the Ohnesorge numbers (Oh), using Eq.13 and Eq. 14, respectively,

$$
\begin{aligned}
& E o=\frac{\Delta \rho g L^{2}}{\gamma} \\
& O h=\frac{\mu}{\sqrt{\rho \gamma L}}
\end{aligned}
$$


where $\Delta \rho$ is the difference in density between the two phases, $g$ the gravitational acceleration, $\mu$ is the dynamic viscosity of the liquid, $\rho$ is the density of the liquid, $\gamma$ is the surface tension and $L$ is the diameter of the pore. The resulting flow map is presented in Figure 23.

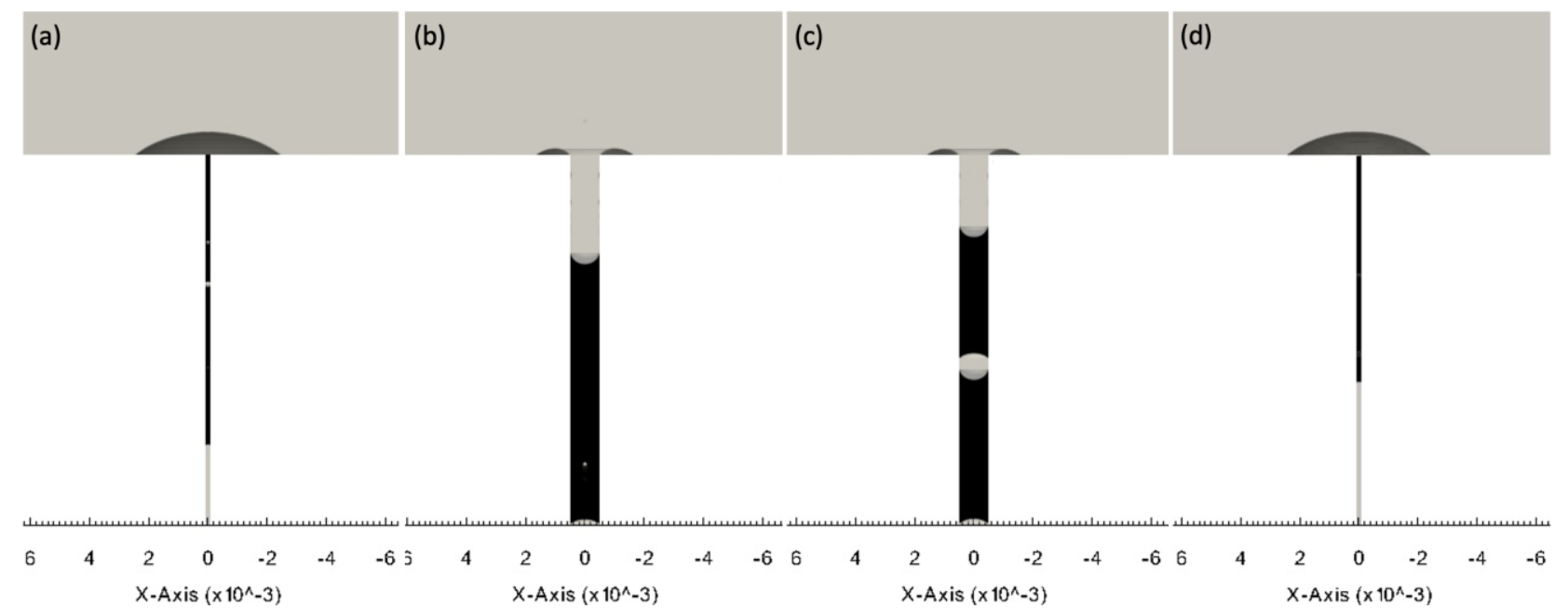

Fig. 22. Indicative snapshots from the last stages of the identified flow regimes; (a) bubble entrapment, (b) bubble entrapment and cleaving, (c) slug-plug flow and cleaving and (d) no entrapment.
0.001
0.01
0.1

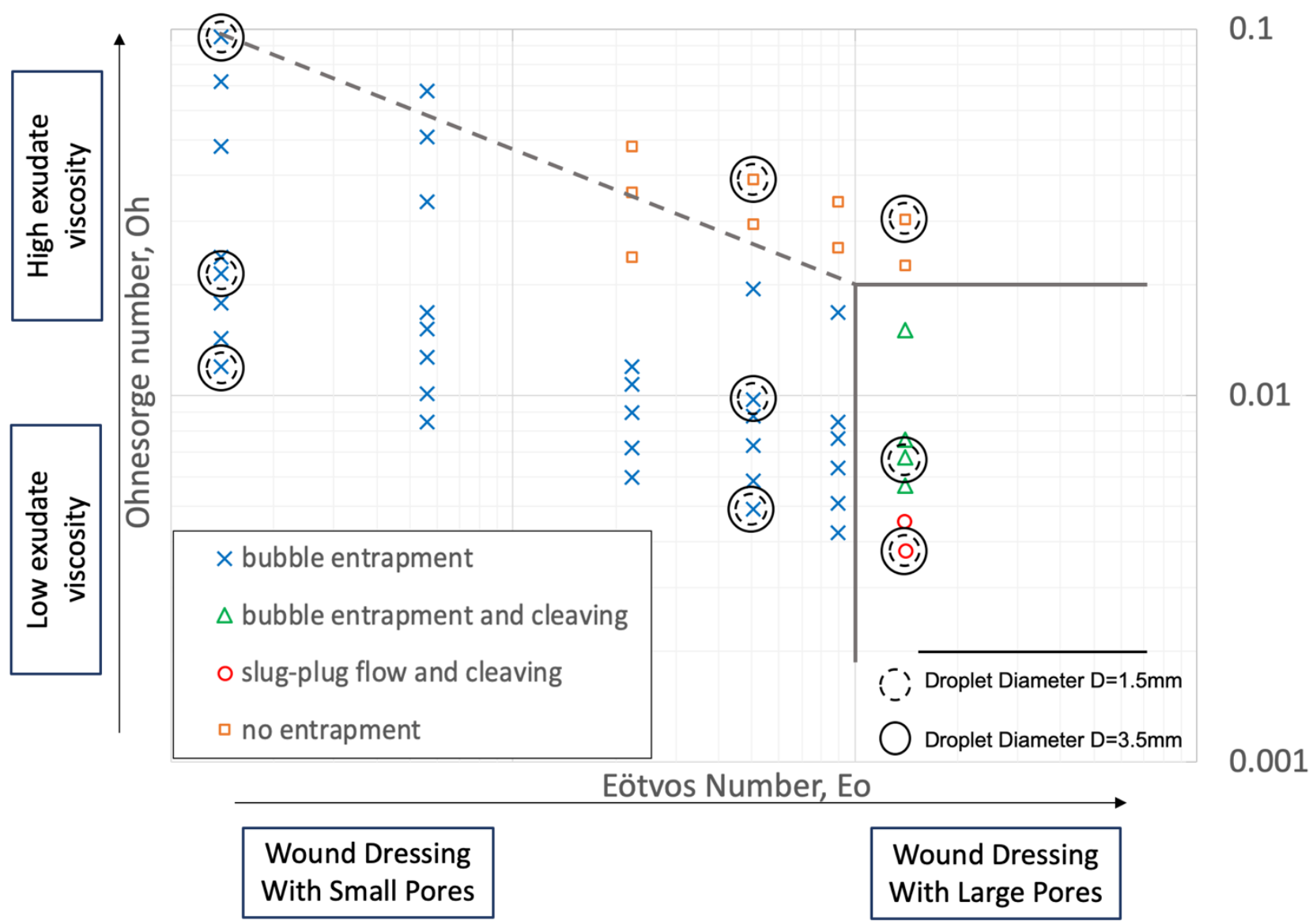

Figure 23. Flow map with different regimes; Ohnesorge Number versus Eötvos Number.

As it can be seen, the previously identified, presented and discussed flow regimes are grouped into a flow map with well-defined boundaries. For the examined dimensionless number ranges "bubble entrapment" seems to be the prevailing regime, ranging between $0.0015<$ Eo $<0.09$ and $0.004<\mathrm{Oh}<0.095$, with "no entrapment" appearing for $0.012<$ Eo $<0.15$ and $0.022<\mathrm{Oh}<0.05$, "bubble entrapment and cleaving" appearing for Eo $>0.15$ and $0.0055<\mathrm{Oh}<0.016$ and "slug-plug 
flow" appearing for Eo $>0.15$ and Oh $<0.0035$. It should be highlighted here that the dotted and solid line circles mark the cases with the same Eo and Oh numbers but for initial droplet diameters of $1.5 \mathrm{~mm}$ and $3.5 \mathrm{~mm}$, respectively. This indicates that the variation of the droplet initial diameter does not affect the resulting flow regimes.

It is quite clear from the diagram of Figure 23, that there seems to be a critical Eo number of $E o=0.1$. For $E o \leq 0.1$, irrespectively of the Oh number the prevailing flow regimes are either "no entrapment" or "bubble entrapment" that constitute the desired condition. For Eo $>0.1$, apart from the "no entrapment" regime that it is desired, the "bubble entrapment and cleaving" and the "slugplug flow and cleaving" flow regimes that are not desired might also appear, depending on the viscosity value of the wound exudate. Hence, solving Equation (13) with respect to the characteristic length (L), which in effect is the pore diameter $d_{\text {pore }}$, the following condition is derived:

$$
d_{\text {pore }} \leq \sqrt{\frac{0.1 \gamma}{\Delta \rho g}}
$$

The above condition can constitute a valuable indication for the most appropriate wound dressing class selection (selecting the appropriate wound dressing porosity), through a measurement of the physical properties for each particular type of wound exudate.

\section{Conclusions}

The ultimate aim of the present investigation is to understand the effect of pore size and liquid viscosity on the main absorption characteristics within porous domains, which are of direct interest for wound dressing applications that constitute the main motivation for this study. For this purpose, a simplified analogue of the proposed problem, considering non-impacting, quasi-sessile droplets that are absorbed by capillary action within single pores, is investigated for the first time, using numerical simulations. In more detail, an enhanced, user-defined, Volume Of Fluid (VOF) model that has been developed in OpenFOAM is used. Despite the fact that the utilised enhanced numerical simulation framework, has been widely validated in the past by the authors for various applications of droplet and bubble dynamics, a further validation using literature available experiments on droplet impacting single cylindrical pores, is initially conducted. Then, two different series of parametric numerical simulations, investigating the effects of pore size and liquid viscosity as well as the effect of initial droplet diameter, are conducted, but for quasi-sessile drops in order to study the absorption characteristics without any initial momentum as in the case of impacting droplets in previous investigations. From the overall presentation and analysis of the numerical results the following worth mentioning specific conclusions are drawn:

- Distinct droplet absorption rates have been identified and specific relationships have been derived, using best-fit lines, that can predict the absorption rates for particular values of pore size (i.e. wound dressing type) and liquid viscosity (i.e. wound exudate class). These distinct droplet absorption rates are directly linked with four different droplet evolution regimes: a) bubble entrapment, b) bubble entrapment and cleaving, c) slug-plug flow and cleaving and, d) no entrapment.

- Through dimensional analysis, these distinct flow regimes are grouped into a flow map with well-defined boundaries. For the examined ranges the "bubble entrapment" regime is the prevailing one, ranging between $0.0015<$ Eo $<0.09$ and $0.004<$ Oh $<0.095$, with "no entrapment" regime appearing for $0.012<$ Eo $<0.15$ and $0.022<\mathrm{Oh}<0.05$, "bubble entrapment and cleaving" regime appearing for Eo $>0.15$ and $0.0055<\mathrm{Oh}<0.016$ and "slug-plug flow" regime appearing for Eo $>0.15$ and $\mathrm{Oh}<0.0035$.

- The initial droplet diameter does not influence significantly the resulting liquid absorption characteristics, apart from cases of small pore size and low viscosity liquids. This is also confirmed by the fact that different initial droplet diameters fall under the same flow regime, for the same Eo and Oh numbers, considering the pore diameter as the characteristic length. 
- Finally, from the mapping of the identified flow regimes, a valuable conditional relationship that links the appropriate pore diameter of the wound dressing with the physical properties of the wound exudate, valid for variable wound exudate viscosities, is derived.

In general, through the present investigation, it is evident that the liquid viscosity as well as the pore diameter are both important controlling parameters as they are directly responsible for the developed liquid absorption regimes and the resulting absorption rates. This is an important finding for wound healing purpose applications. Moreover, it is evident that the proposed numerical simulation framework constitutes an important tool that can provide valuable insight, quantifying in detail the underpinned phenomena for liquid absorption characteristics into porous materials. In the future, the effect of the wettability characteristics on the penetration of a quasi-sessile droplet in a single capillary should also be investigated and the present investigation results should be extended from single to multiple pores or even more complex pore networks. Furthermore, the concept of a liquid film instead of a drop should also be studied in order to move even closer to the targeted application.

\section{Acknowledgments}

Dr. Andredaki would like to thank University of Brighton for the support of the present work through the Rising Stars Initiative 2019-2020 Scheme. Prof. Marengo and Dr. Anastasios Georgoulas would like to thank the financial support from ESA MAP projects TOPDESS and ENCOM 4.

\section{Data Availability Statement}

The data that support the findings of this study are available from the corresponding author upon reasonable request.

\section{References}

[1] BS EN 13726: 2002, “Testing methods for primary wound dressings," British Standard. ICS 11.120.20. .

[2] G. D. WINTER, "Formation of the Scab and the Rate of Epithelization of Superficial Wounds in the Skin of the Young Domestic Pig," Nature, vol. 193, no. 4812, pp. 293-294, 1962.

[3] K. Cutting, "Exudate: Composition and functions," in Trends in Wound Care: Volume III., R. White, Ed. Quay Books, London, 2004.

[4] L. G. Ovington, “Advances in wound dressings," Clin. Dermatol., vol. 25, no. 1, pp. 33-38, 2007.

[5] S. Dhivya, V. V. Padma, and E. Santhini, "Wound dressings - a review," BioMedicine, vol. 5, no. 4, p. 22, Dec. 2015.

[6] A. Sood, M. S. Granick, and N. L. Tomaselli, "Wound Dressings and Comparative Effectiveness Data," Adv. wound care, vol. 3, no. 8, pp. 511-529, Aug. 2014.

[7] Z. T. Piskozub, "The efficiency of wound dressing materials as a barrier to secondary bacterial contamination," Br. J. Plast. Surg., vol. 21, no. 4, pp. 387-401, 1968.

[8] S. Thomas, C. Dawes, and N. Hay, "Wound dressing materials - testing and control," Pharm $J$, no. 228, p. 576, 1982.

[9] S. Thomas, "Surgical Dressings and Wound Management," in Surgical Dressings and Wound Management, Hinesburg, VT: Kestrel Health, 2012, pp. 79-106.

[10] Y. Suzuki et al., "In vivo evaluation of a novel alginate dressing," J. Biomed. Mater. Res., vol. 48, no. 4, p. 522-527, 1999.

[11] K. W. Ng, H. N. Achuth, S. Moochhala, T. C. Lim, and D. W. Hutmacher, "In vivo evaluation of an ultra-thin polycaprolactone film as a wound dressing," J. Biomater. Sci. Polym. Ed., vol. 18, no. 7, pp. 925-938, Jan. 2007.

[12] D. Archana, J. Dutta, and P. K. Dutta, "Evaluation of chitosan nano dressing for wound healing: Characterization, in vitro and in vivo studies," Int. J. Biol. Macromol., vol. 57, pp. 193-203, 2013. 
[13] D. Archana, B. K. Singh, J. Dutta, and P. K. Dutta, "In vivo evaluation of chitosan-PVPtitanium dioxide nanocomposite as wound dressing material," Carbohydr. Polym., vol. 95, no. 1, pp. 530-539, 2013.

[14] P. Aramwit, P. Muangman, N. Namviriyachote, and T. Srichana, "In vitro evaluation of the antimicrobial effectiveness and moisture binding properties of wound dressings," Int. J. Mol. Sci., vol. 11, no. 8, pp. 2864-2874, Aug. 2010.

[15] P. Wu, E. A. Nelson, W. H. Reid, C. V Ruckley, and J. D. S. Gaylor, "Water vapour transmission rates in burns and chronic leg ulcers: influence of wound dressings and comparison with in vitro evaluation," Biomaterials, vol. 17, no. 14, pp. 1373-1377, 1996.

[16] D. McColl, B. Cartlidge, and P. Connolly, "Real-time monitoring of moisture levels in wound dressings in vitro: An experimental study," Int. J. Surg., vol. 5, no. 5, pp. 316-322, 2007.

[17] R. White and K. Cutting, "Modern exudate man-agement: A review of wound treatments," World Wide Wounds, vol. 1.0, 2006.

[18] P. Vowden, E. Bond, and F. Meulenetre, "Managing high viscosity exudate," Wounds Int., vol. 6, no. 1, pp. 14-19, 2015.

[19] K. Cutting, "Wound exudate: composition and functions," Br. J. Community Nurs., vol. 8, no. 3, pp. 4-9, 2013.

[20] H. K. Navaz et al., "Sessile droplet spread into porous substrates-Determination of capillary pressure using a continuum approach," J. Colloid Interface Sci., vol. 325, no. 2, pp. 440-446, 2008.

[21] B. Markicevic, H. Li, Y. Sikorski, A. R. Zand, M. Sanders, and H. K. Navaz, "Infiltration time and imprint shape of a sessile droplet imbibing porous medium," J. Colloid Interface Sci., vol. 336, no. 2, pp. 698-706, 2009.

[22] X. Frank and P. Perré, "Droplet spreading on a porous surface: A lattice Boltzmann study," Phys. Fluids, vol. 24, no. 4, p. 042101, Apr. 2012.

[23] M. Denesuk, G. L. Smith, B. J. J. Zelinski, N. J. Kreidl, and D. R. Uhlmann, "Capillary Penetration of Liquid Droplets into Porous Materials," J. Colloid Interface Sci., vol. 158, no. 1, pp. 114-120, 1993.

[24] R. Sadeghi, M. S. Shadloo, M. Hopp-Hirschler, A. Hadjadj, and U. Nieken, "Threedimensional lattice Boltzmann simulations of high density ratio two-phase flows in porous media," Comput. Math. with Appl., vol. 75, no. 7, pp. 2445-2465, 2018.

[25] M. Choi, G. Son, and W. Shim, "A level-set method for droplet impact and penetration into a porous medium," Comput. Fluids, vol. 145, pp. 153-166, 2017.

[26] T. C. de Goede et al., "Droplet impact of Newtonian fluids and blood on simple fabrics: effect of fabric pore size and underlying substrate," 2020.

[27] A. Koponen, M. Kataja, and J. Timonen, "Permeability and effective porosity of porous media," Phys. Rev. E, vol. 56, no. 3, pp. 3319-3325, Sep. 1997.

[28] J. Kozeny, "Ueber kapillare Leitung des Wassers im Boden.," Sitzungsber Akad. Wiss., vol. 136, no. 2a, pp. 271-306, 1927.

[29] P. C. Carman, "Fluid flow through granular beds," Trans. Inst. Chem. Eng. London, vol. 15, pp. $150-166,1937$.

[30] P. C. Carman, "Flow of gases through porous media," Butterworths, London, 1956.

[31] B. Yu and P. Cheng, "A fractal permeability model for bi-dispersed porous media," Int. J. Heat Mass Transf., vol. 45, no. 14, pp. 2983-2993, 2002.

[32] V. Kogan, E. Johnson, P. Schumacher, and B. Shroy, "Capillary flow of liquid under droplet impact conditions," in L22nd European Conference on Liquid Atomization Spray Systems, 2008.

[33] A. Delbos, E. Lorenceau, and O. Pitois, "Forced impregnation of a capillary tube with drop impact," J. Colloid Interface Sci., vol. 341, no. 1, pp. 171-177, 2010.

[34] H. Ding and T. G. Theofanous, "The inertial regime of drop impact on an anisotropic porous substrate," J. Fluid Mech., vol. 691, pp. 546-567, 2012. 
[35] S. M. Lee et al., "Physical, morphological, and wound healing properties of a polyurethane foam-film dressing.," Biomater. Res., vol. 20, p. 15, 2016.

[36] T. T. V. Phan, T.-C. Huynh, and J. Oh, "Photothermal Responsive Porous Membrane for Treatment of Infected Wound.," Polymers (Basel)., vol. 11, no. 10, Oct. 2019.

[37] “OpenFOAM, The Open Source CFD Toolbox, User Guide," no. v. 2.2.1, 2013.

[38] J. . Brackbill, D. . Kothe, and C. Zemach, "A continuum method for modeling surface tension,” J. Comput. Phys., vol. 100, no. 2, pp. 335-354, Jun. 1992.

[39] A. Georgoulas, P. Koukouvinis, M. Gavaises, and M. Marengo, "Numerical investigation of quasi-static bubble growth and detachment from submerged orifices in isothermal liquid pools: The effect of varying fluid properties and gravity levels," Int. J. Multiph. Flow, vol. 74, pp. 59-78, 2015.

[40] S. Kistler, "Hydrodynamics of wetting," Wettability, vol. 6, no. Marcel Dekker, New York, pp. 311--430, 1993.

[41] K. Vontas, M. Andredaki, A. Georgoulas, K. S. Nikas, and M. Marengo, "Numerical Investigation of Droplet Impact on Smooth Surfaces with Different Wettability Characteristics: Implementation of a dynamic contact angle treatment in OpenFOAM," in 28th European Conference on Liquid Atomization and Spray Systems, 2017.

[42] K. Vontas et al., "Droplet Impact on Suspended Metallic Meshes: Effects of Wettability, Reynolds and Weber Numbers," Fluids, vol. 5, no. 2, p. 81, 2020.

[43] C. Antonini, A. Amirfazli, and M. Marengo, "Drop impact and wettability: From hydrophilic to superhydrophobic surfaces," Phys. fluids, vol. 24, no. 10, p. 102104, 2012.

[44] M. Miyama, Y. Yang, T. Yasuda, T. Okuno, and H. K. Yasuda, "Static and Dynamic Contact Angles of Water on Polymeric Surfaces," Langmuir, vol. 13, no. 20, pp. 5494-5503, Oct. 1997.

[45] G. Mulder, "Quantifying wound fluids for the clinician and researcher," Ostomy Wound Manag., vol. 40, no. 8, pp. 66-69, 1994.

[46] E. W. Washburn, “The Dynamics of Capillary Flow," Phys. Rev., vol. 17, no. 3, pp. $273-$ 283, Mar. 1921. 


\section{Open End}

Simulation Type:

2D Axisymmetric

( $5^{0}$ Wedge)

Grid Type:

Uniform, Structured, Hexahedral Cells

Total No. of Cells:

253,125 (for Pore radius $0.1 \mathrm{~mm}$ )

256,875 (for Pore radius $0.22 \mathrm{~mm}$ ) 265,625 (for Pore radius $0.5 \mathrm{~mm}$ )

Cell Size: $2 \mu \mathrm{m}$

Walls

$\theta_{\mathrm{c}}=30^{\circ}$

$\theta_{\mathrm{a}}=48^{0}$

$\theta_{\mathrm{r}}=5^{0}$
Wall

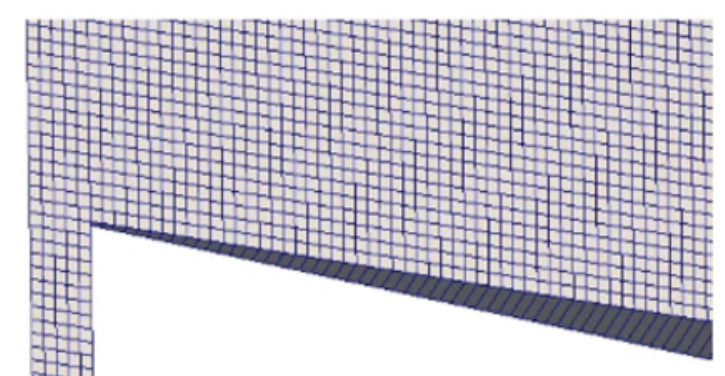




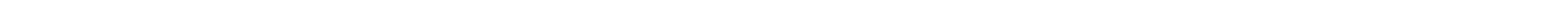




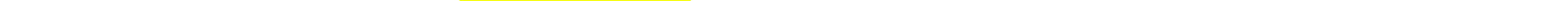




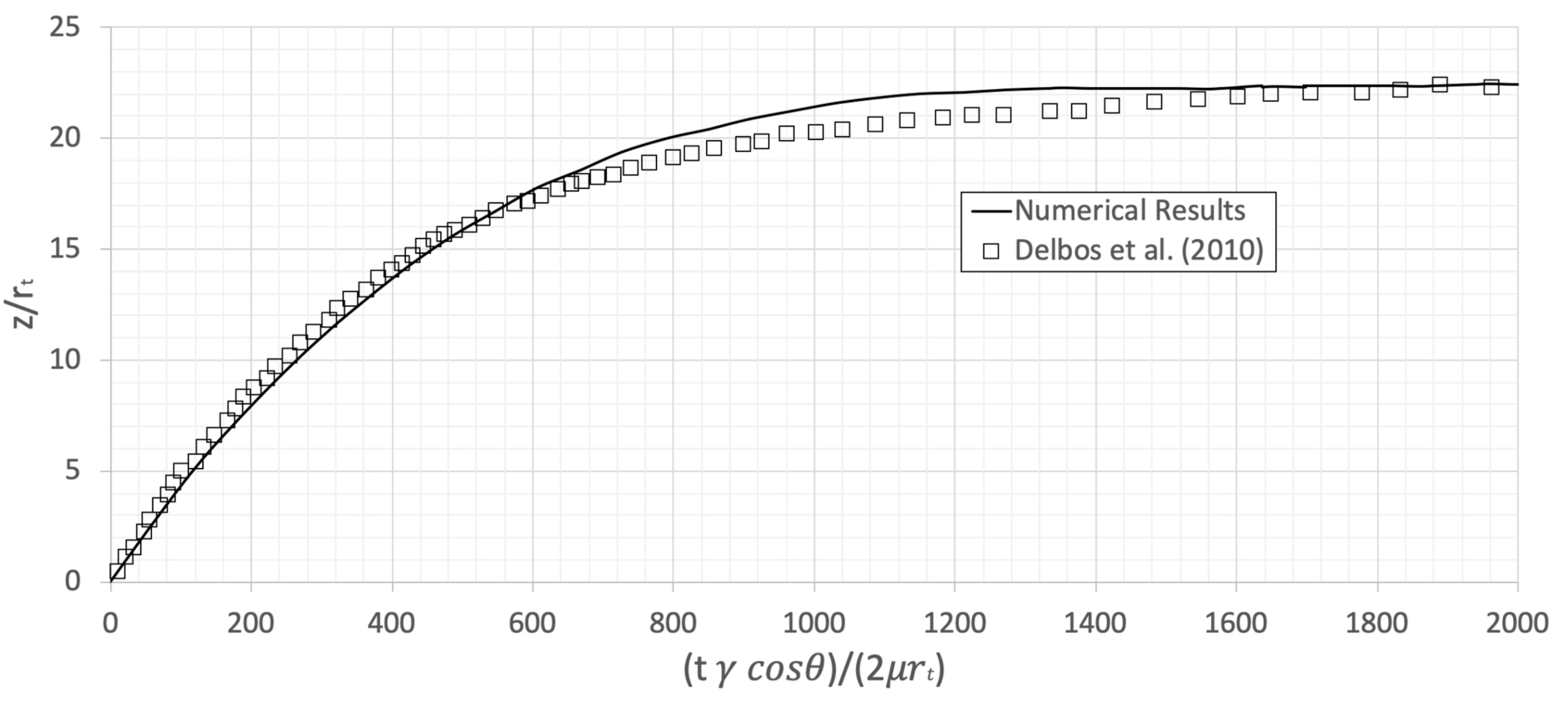




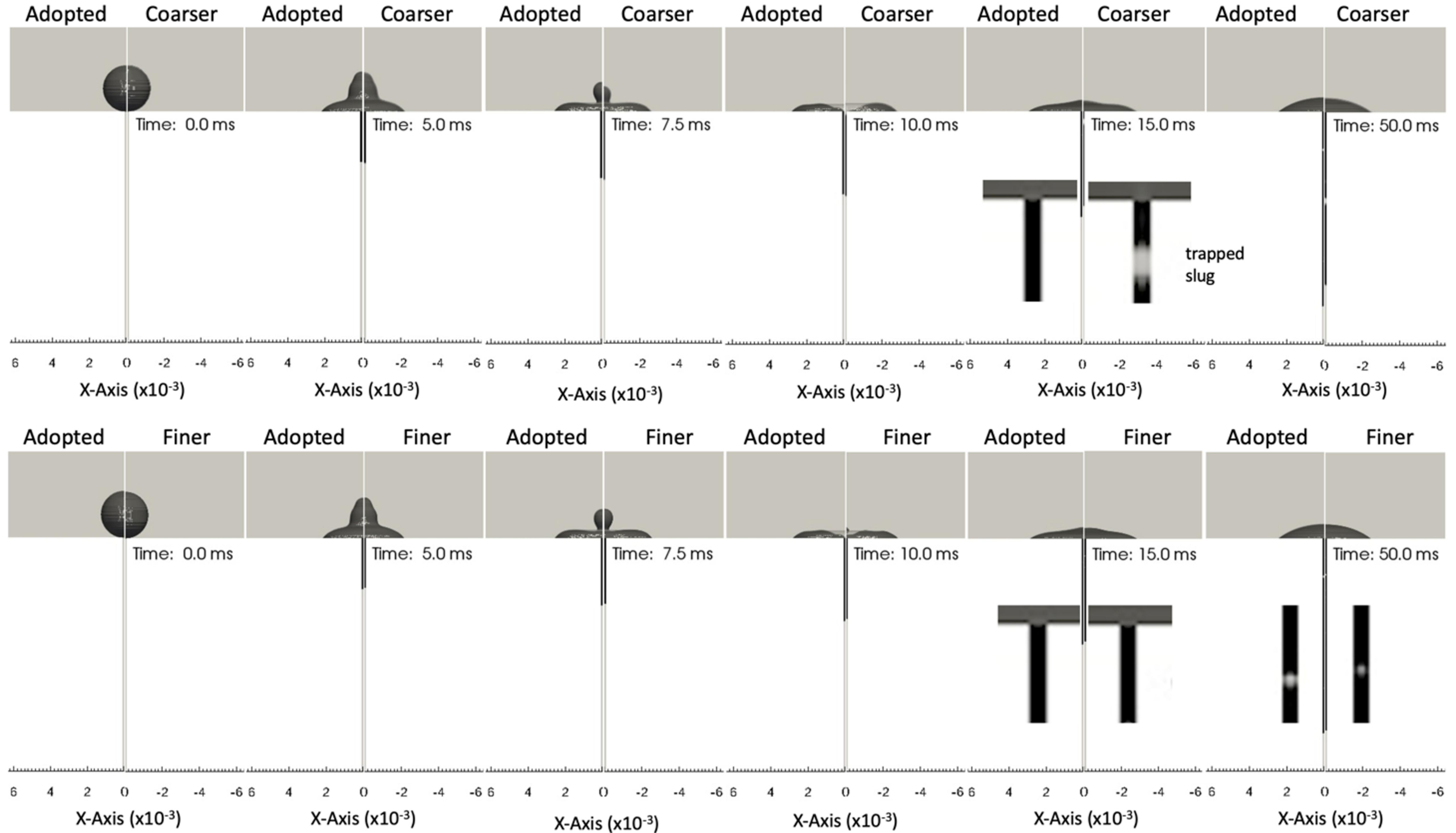




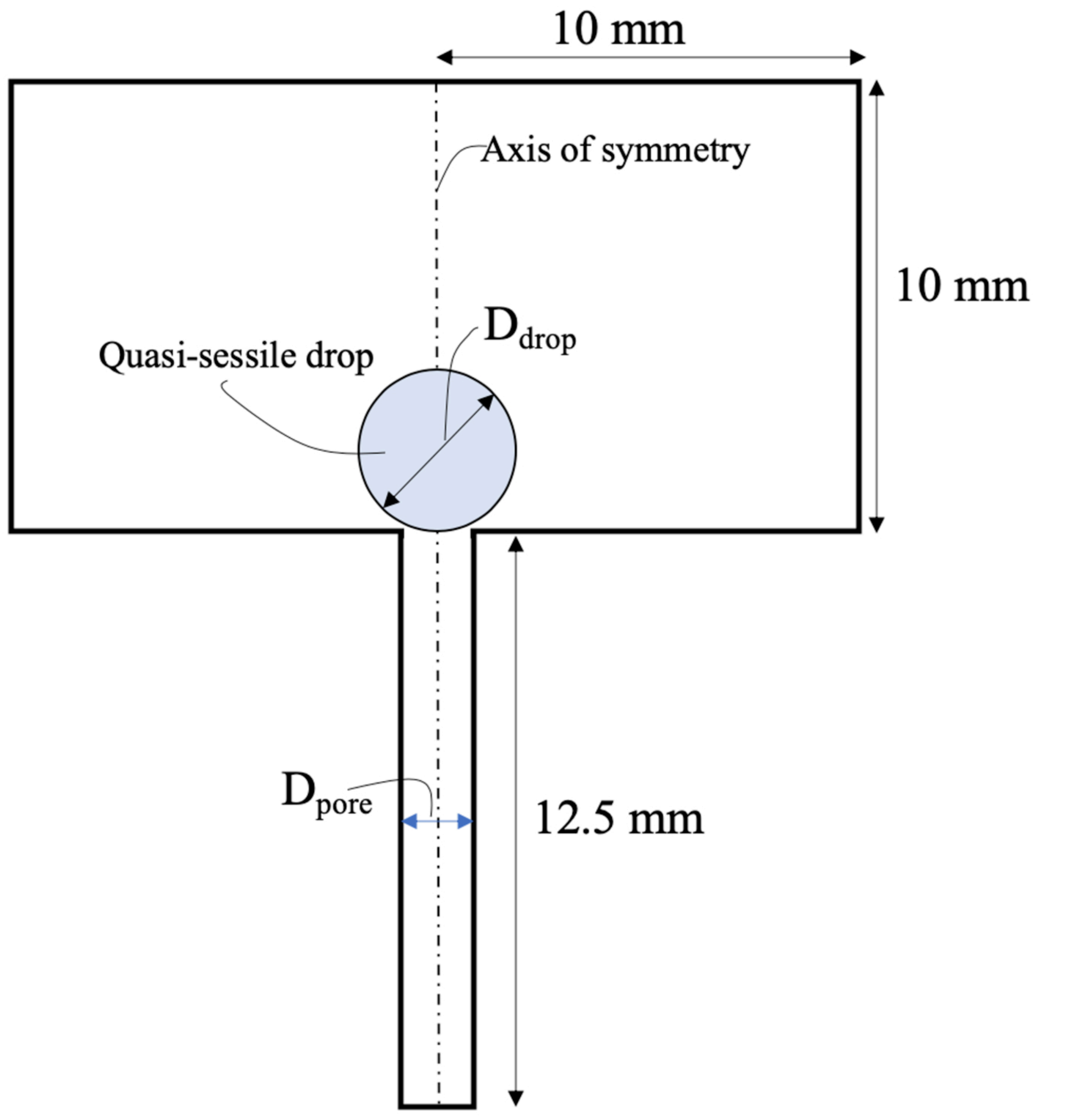




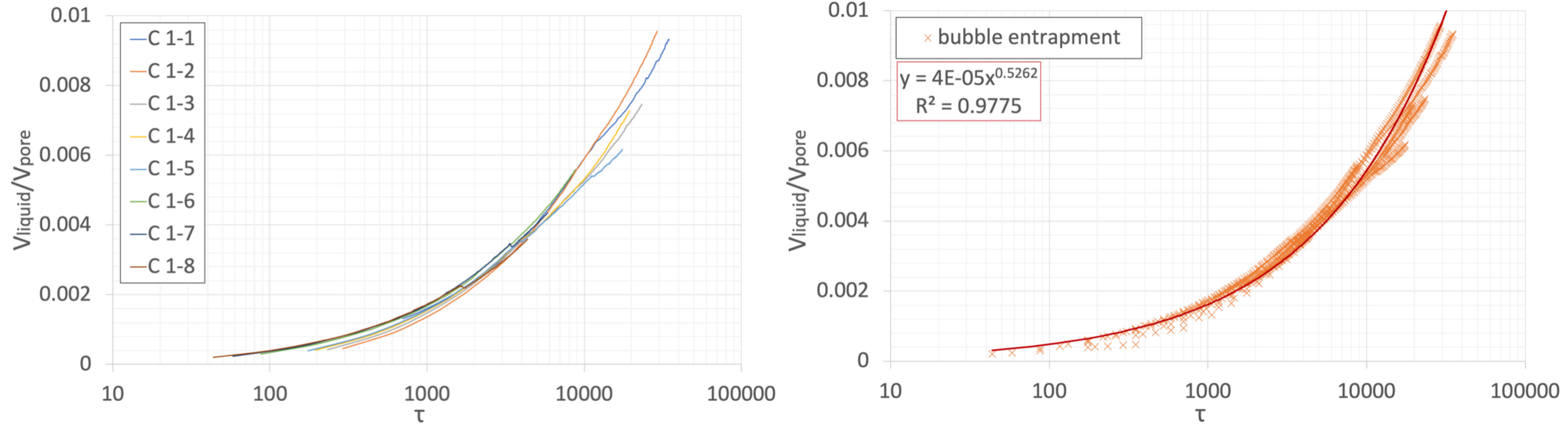




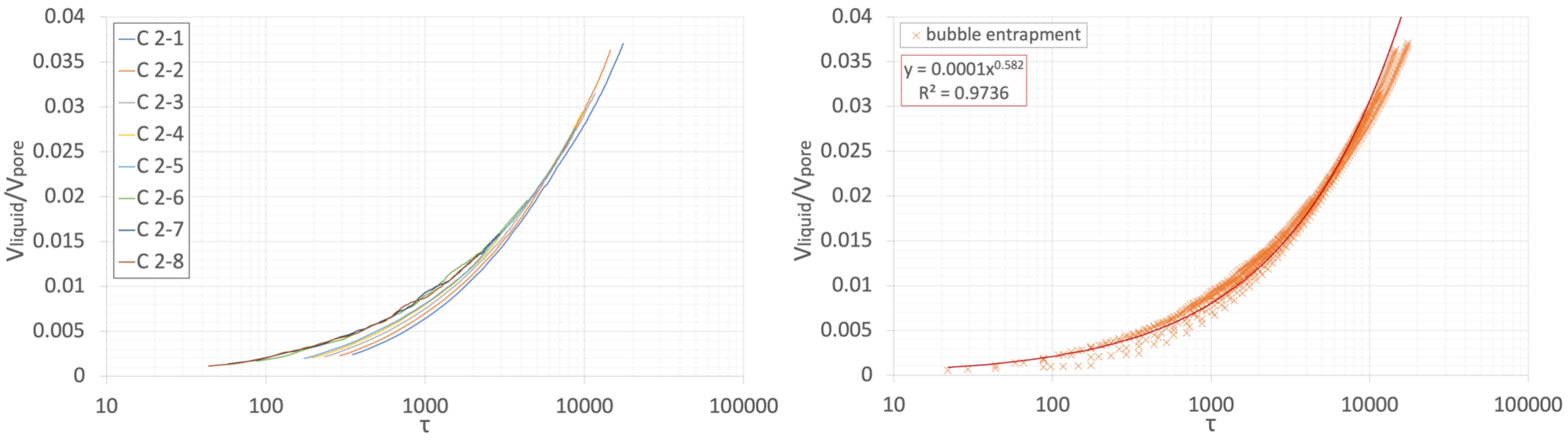




\section{Time: $5.0 \mathrm{~ms}$}

\section{Time: $10.0 \mathrm{~ms}$}

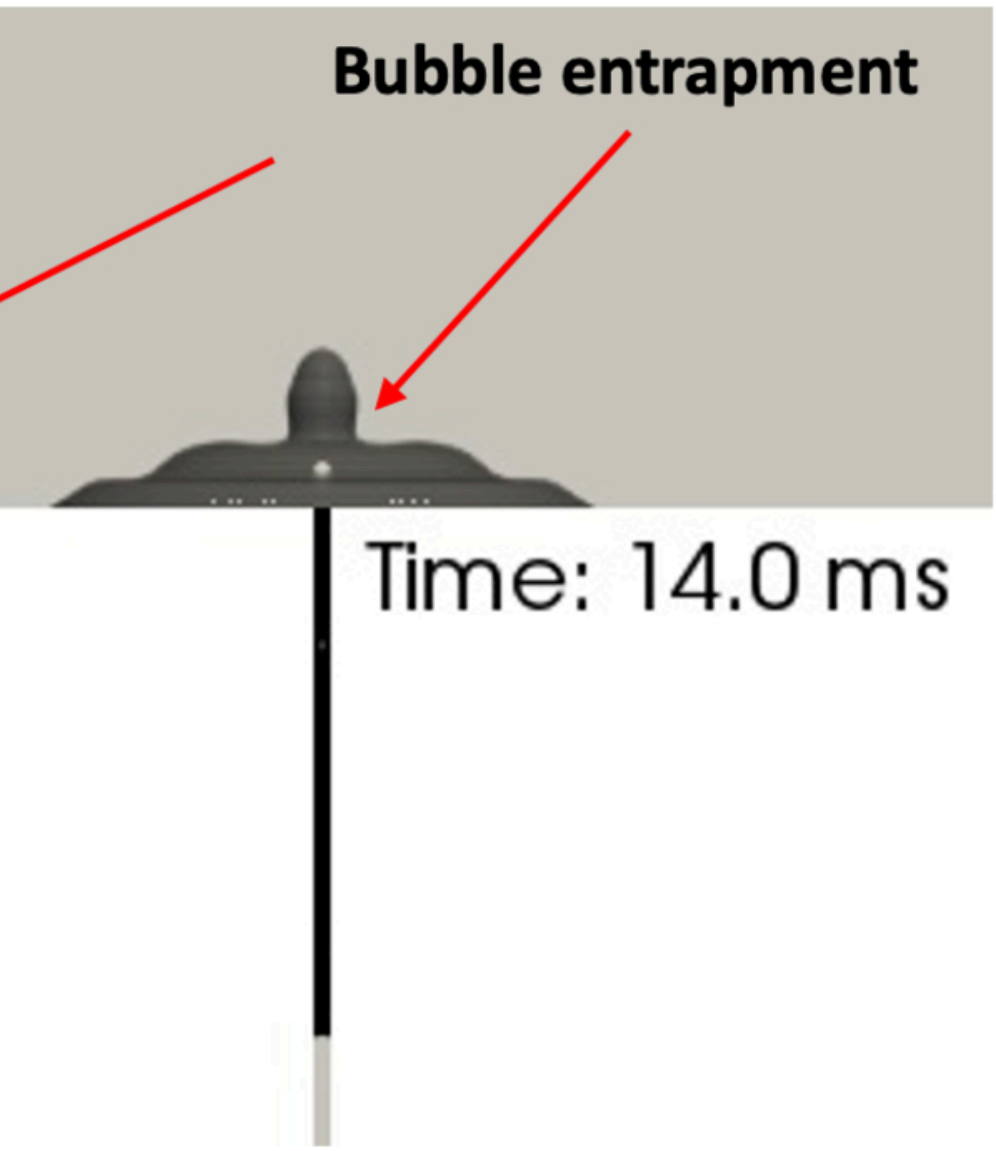

\section{Disintegration of} entrapped bubble in smaller bubbles as it penetrates the pore

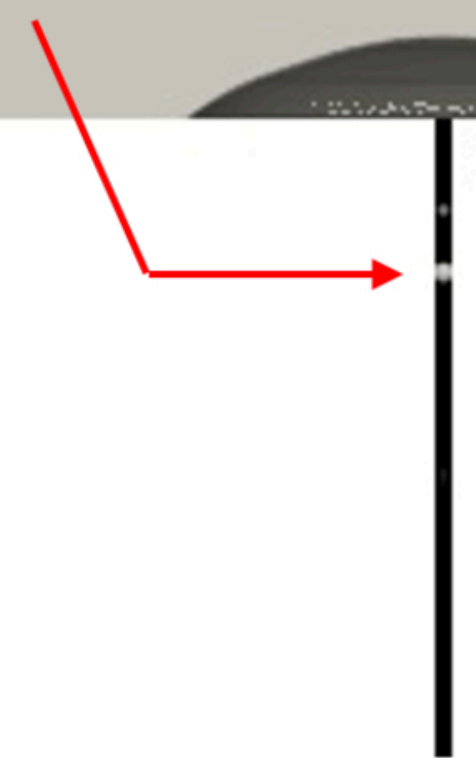

Time: $25.0 \mathrm{~ms}$ 


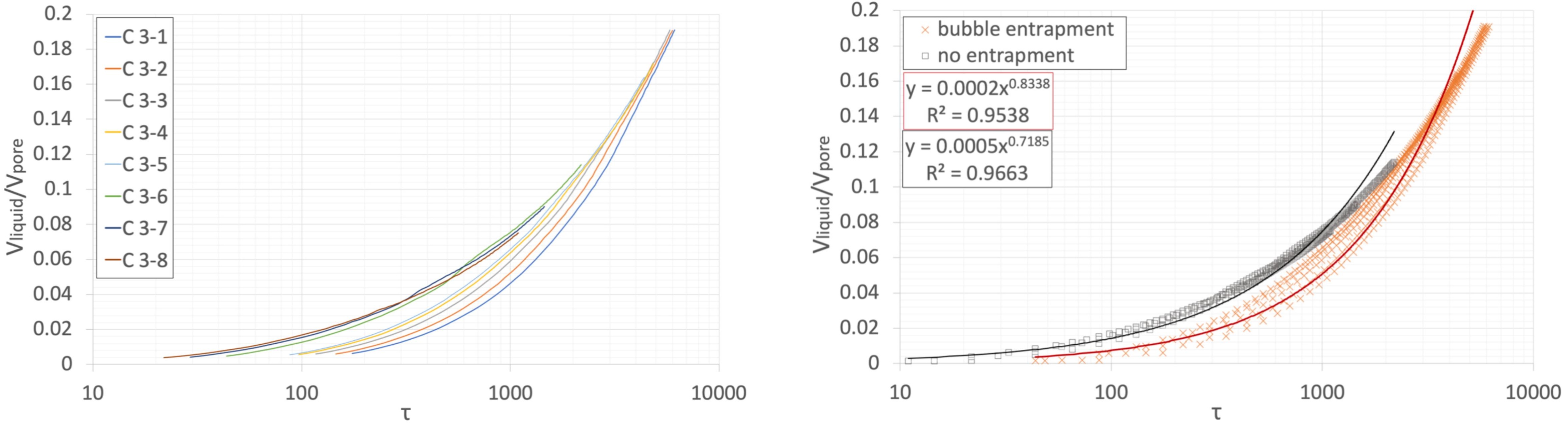




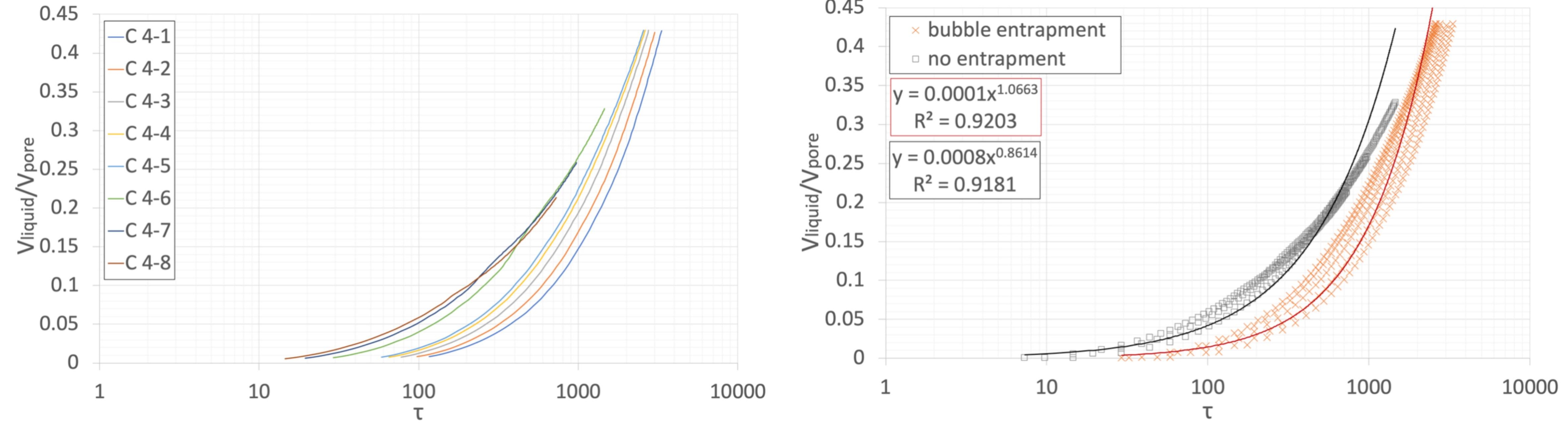




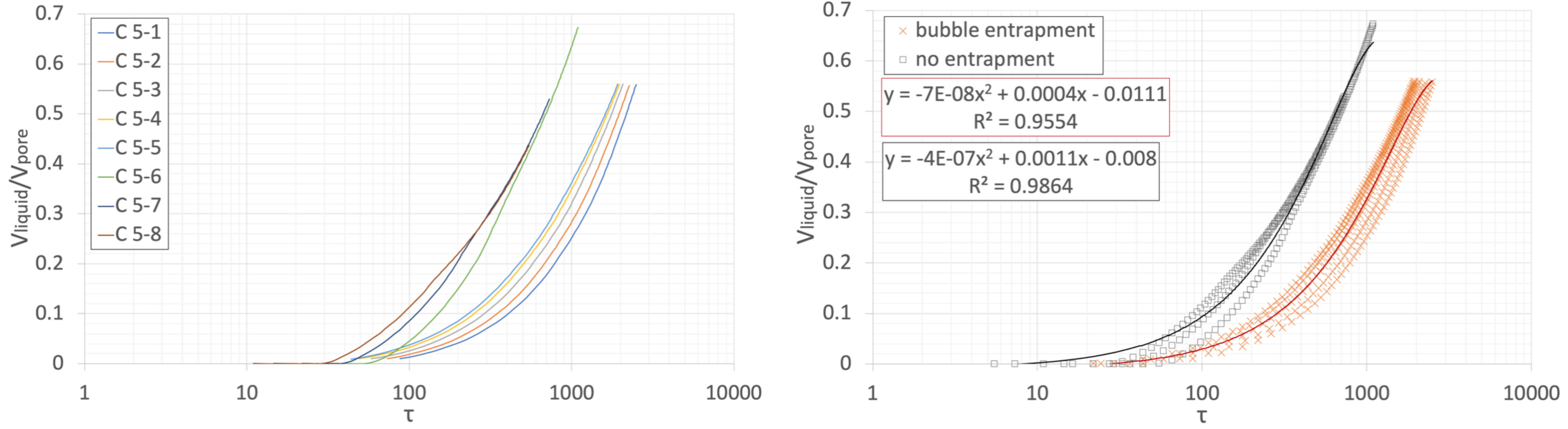




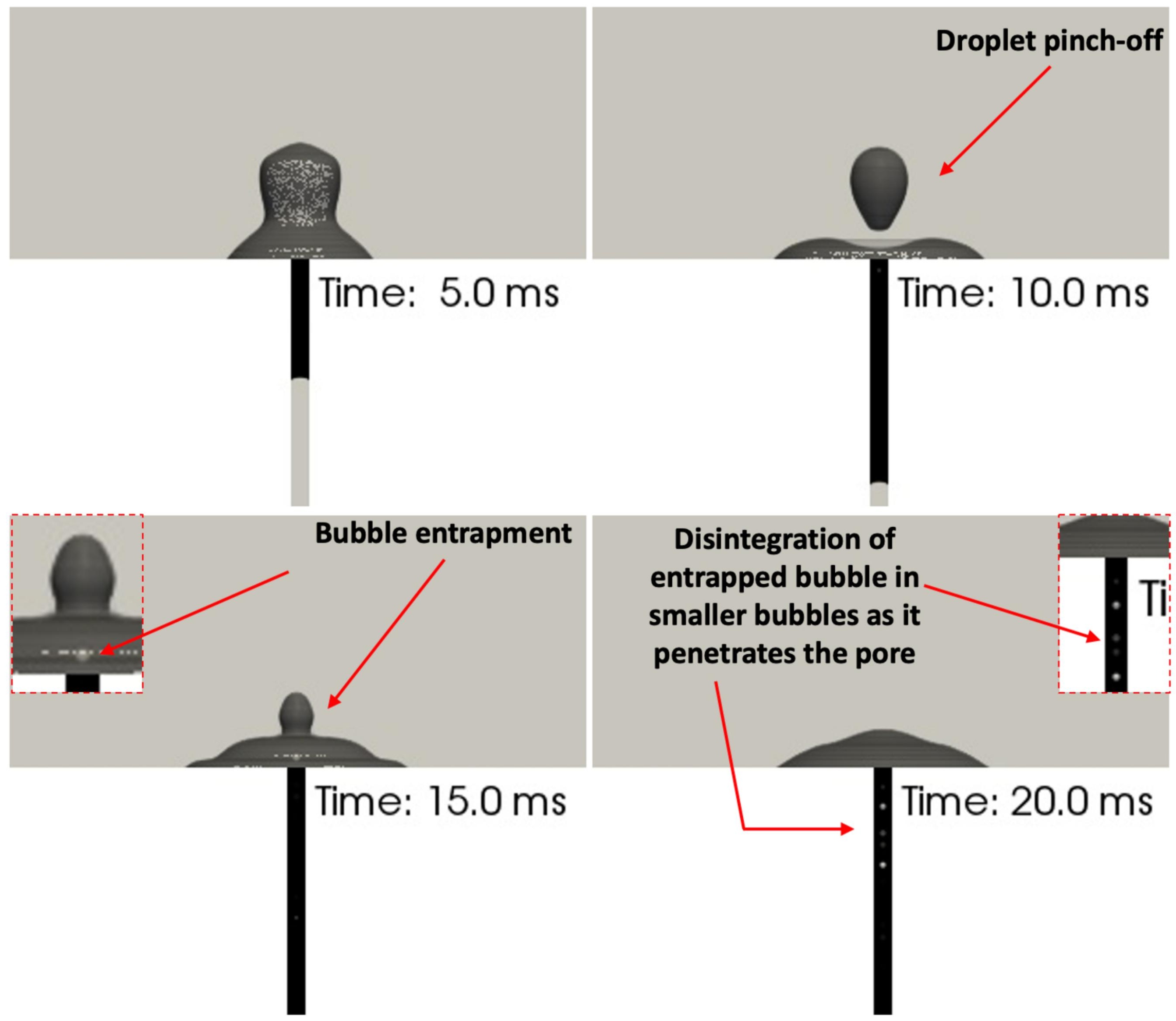




\section{No droplet pinch-off}

Time: $5.0 \mathrm{~ms}$

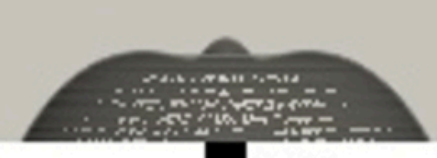

Time: $10.0 \mathrm{~ms}$

No bubble entrapment

Time: $10.5 \mathrm{~ms}$

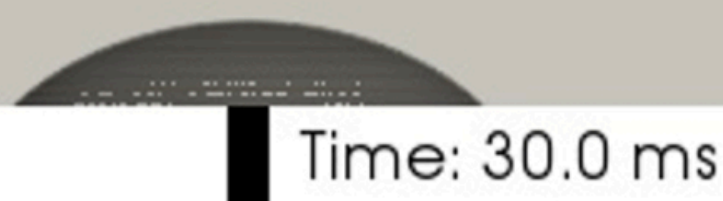




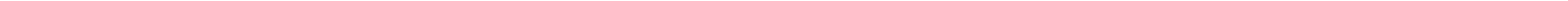


Time: $12.0 \mathrm{~ms}$

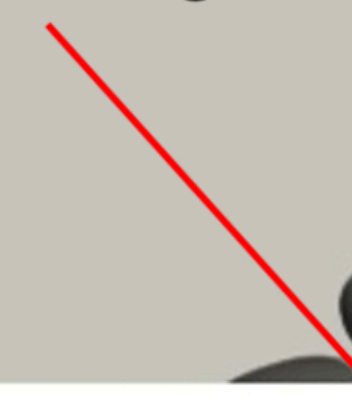

Time: $15.0 \mathrm{~ms}$
Air plug

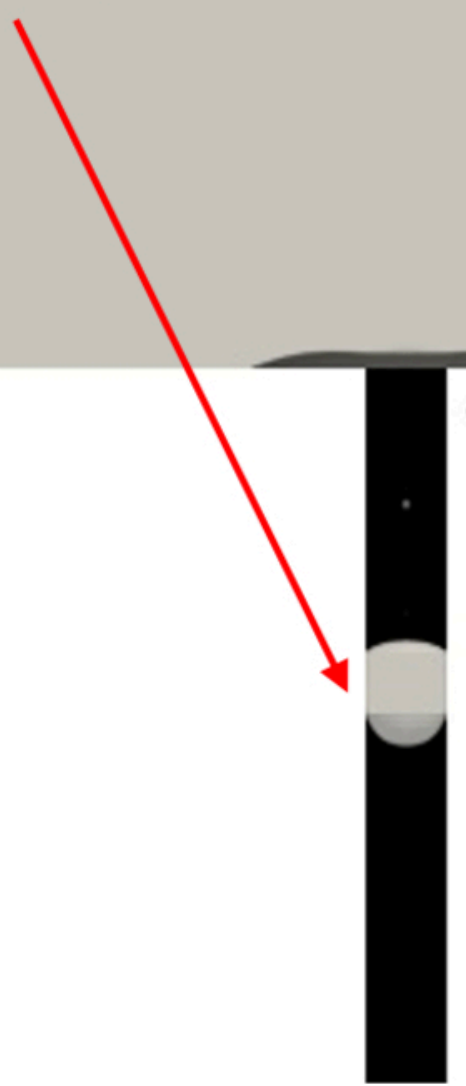

\section{$2^{\text {nd }}$ cleaving}

Time: $25.0 \mathrm{~ms}$
Time: $37.5 \mathrm{~ms}$ 
Droplet pinch-off

Bubble entrapment

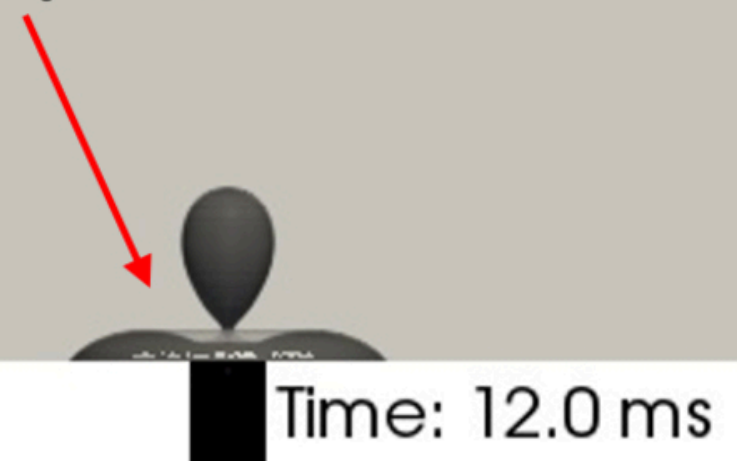

Time: $15.0 \mathrm{~ms}$

Cleaving

Time: $20.0 \mathrm{~ms}$

Time: $35.0 \mathrm{~ms}$ 


\section{No droplet pinch-off}

Time: $10.0 \mathrm{~ms}$

Time: $13.0 \mathrm{~ms}$

No bubble entrapment

Time: $15.0 \mathrm{~ms}$

Time: $30.0 \mathrm{~ms}$ 


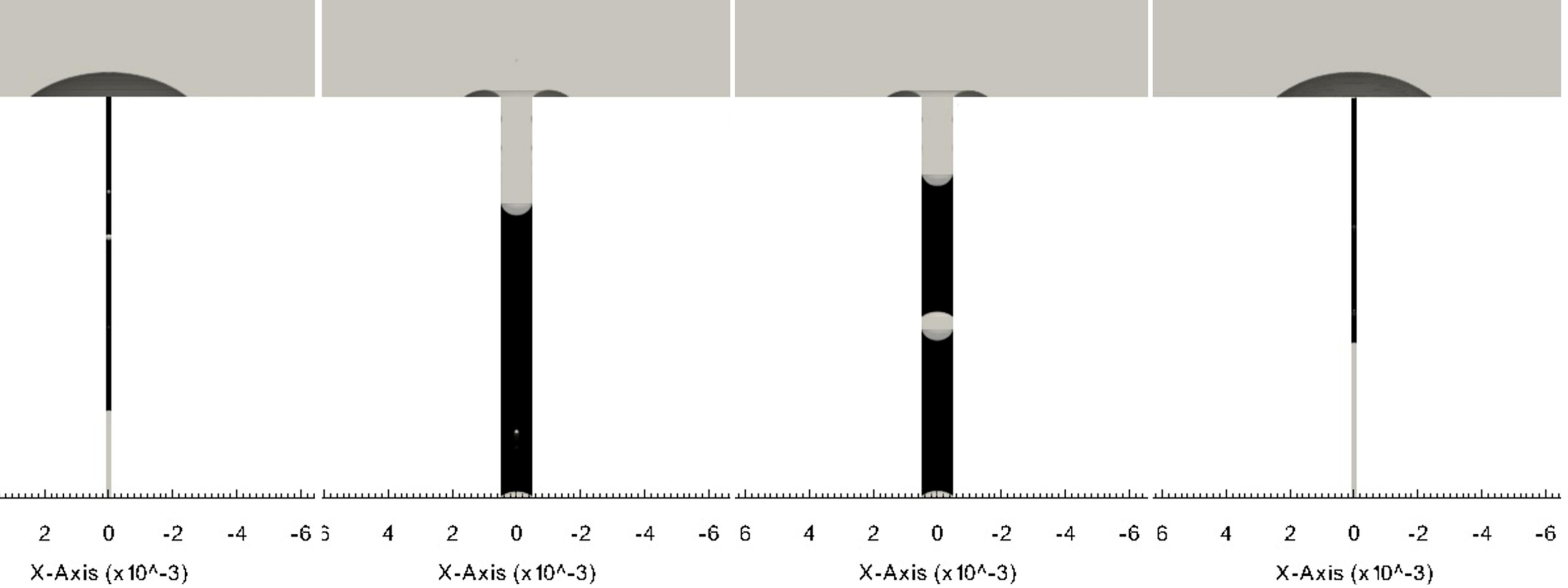




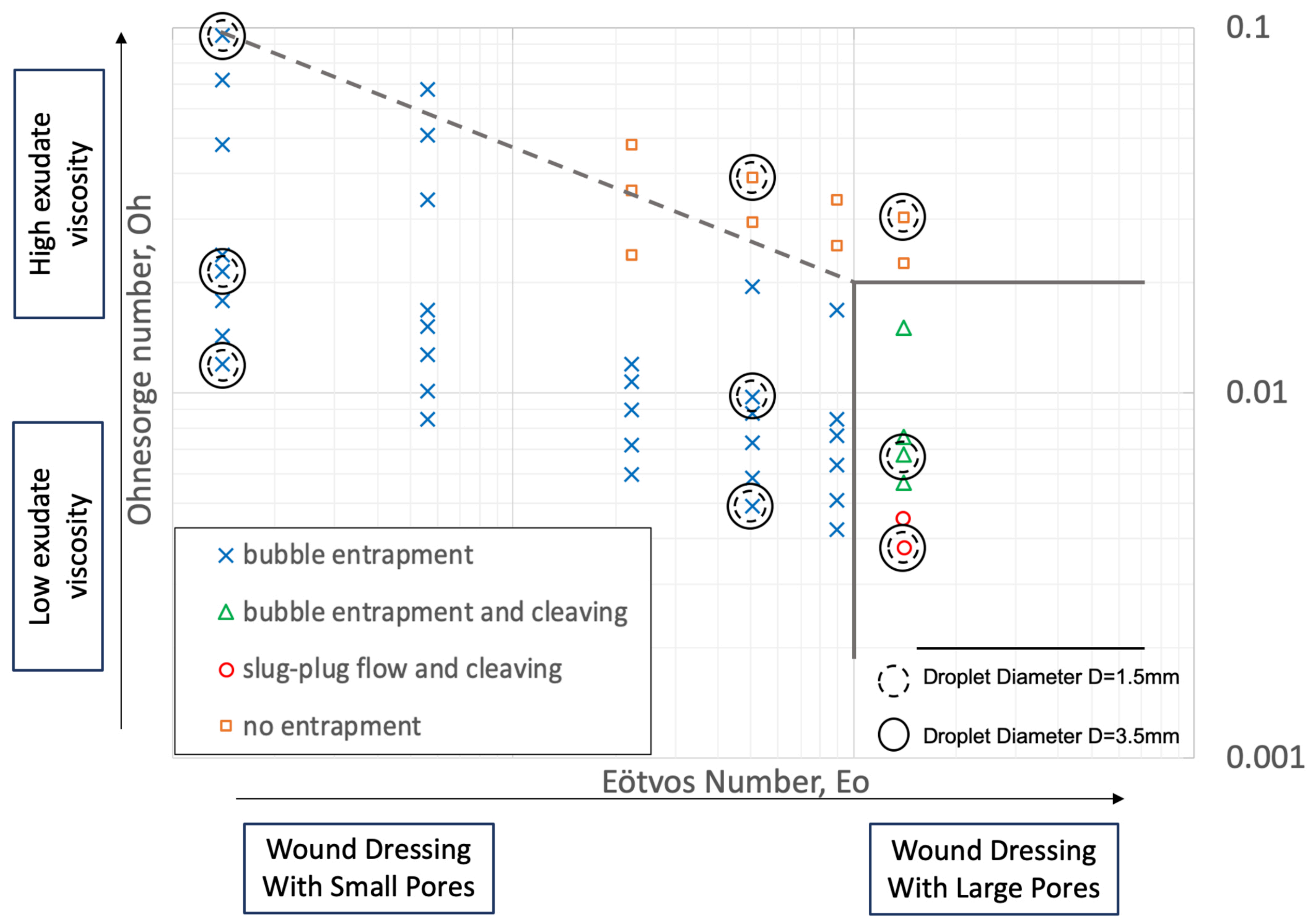

